\title{
DESCRIPTION ET INTERPRÉTATION DES ÉCAILLES DE TRUITES DEMER (SALMO TRUTTA L.) DE DEUX RIVIĖRES DE BASSE-NORMANDIE : L'ORNE ET LA TOUQUES
}

\author{
A. RICHARD (1), J.L. BAGLINIERE (2)
}

(1) Conseil Supérieur de la Pêche - Délégation Régionale $n^{\circ} 2-84$, Rue de Rennes 35510 CESSON SEVIGNE (FRANCE)

(2) Laboratoire d'Écologie Hydrobiologique - INRA - 65, Rue de St Brieuc - 35042 RENNES CEDEX (FRANCE)

\section{RESUME}

Les écailles de plus de 10.000 adultes de truites de mer capturés principalement par piégeage à la remontée sur l'Orne et la Touques (Basse-Normandie) ont été examinées.

L'interprétation de ces écailles et leur validation par la recapture d'individus marqués a permis de :

- distinguer trois phases de croissance de l'écaille caractéristiques d'un migrateur amphibiotique à nos latitudes: rivière, transition, mer.

- déterminer relativement facilement l'âge d'eau douce des poissons en raison d'une bonne croissance des juvéniles permettant d'identifier correctement les bandes hivernales.

- définir quatre types de poissons à partir de l'âge de mer de première reproduction.

- caractériser la marque de reproduction qui se singularise chez cette espèce à frais multiples par l'importance de l'érosion de l'écaille dans sa partie postérieure. Cette marque est d'autant plus prononcée que le poisson a un âge marin élevé à la première reproduction.

- déceler des structures particulières et propres à ces poissons en liaison avec des comportements migratoires variés lors du stade post-smolt.

Mots-clés : Truite de mer, écailles, détermination d'âge, rivière.

\section{DESCRIPTION AND INTERPRETATION OF SEA-TROUT (SALMO TRUTTA L.) SCALES FROM TWO RIVERS IN LOWER-NORMANDY : THE RIVERS ORNE AND TOUQUES}

\section{SUMMARY}

Scales of more 10.000 sea-trout (Salmo trutta L.) adults mainly trapped in the R. Orne and $R$. Touques (Lower Normandy) were analysed. Their interpretation and the validation of scales structures by the recapture of tagged fish have shown:

- a typical distinction between growth zones on the scale for an amphibiotic migratory fish in that latitude: freshwater, run-out and sea growth.

- an easy determination of the freshwater age because of a high growth during the juvenile stage resulting in an accurate identification of winter bands.

- the definition of four sea-trout types according to the sea age at the first reproduction period.

- a spawning mark characterized by a large erosion at the posterior edge of the scale in this multispawner fish. The erosion of the scale increases with the sea age at the first reproduction.

- the existence of particular structures related to various migratory behaviours during post-smolt stage.

Keywords : Sea-trout, scales, determination of the age, river.

\section{INTRODUCTION}

A nos latitudes, les écailles représentent le premier matériel généralement utilisé pour caractériser l'âge des salmonidés (saumon et truite), (BAGLINIERE et LE LOUARN, 1987), qu'il s'agisse d'individus isolés (captures "exceptionnelles" ou par pêche à la ligne) ou de contingents contrôlés plus régulièrement dans des stations de capture.

Si la méthode scalimétrique est utilisée depuis fort longtemps (DAHL, 1909; ROULE, 1920 ; JARVI et MENZIES, 1936 ; VIBERT, 1950; BACKIEL et SYCH, 1958), les critères d'interprétation des 
écailies n'ont fait que récemment l'objet de standardisation et ce uniquement chez le saumon atlantique (Salmo salar L.), d'abord au niveau international (ANONYME, 1984 et 1988) puis au niveau national (BAGLINIERE, 1985).

Bien que la scalimétrie ait été largement utilisée pour caractériser les populations de truite de mer (Salmo trutta L.) à l'étranger (ALM, 1950; SYCH, 1967 a; JENSEN, 1968; CHELKOWSKI, 1969; ZARNECKI, 1973; PRATTEN et SHEARER, 1983; JOHNSSON, 1985), peu de travaux se sont intéressés à l'interprétation des écailles en montrant la bonne fiabilité de cette méthode de détermination (ZARNECKI, 1960 ; CHRZAN, 1962 ; SYCH, 1967b).

En France, aucun travail de ce type n'avait été réalisé sur la truite de mer jusqu'à présent à l'exception de celui de BEALL et DAVAINE (1988) dans les îles Kerguelen.

En 1981, une étude sur la biologie de la truite de mer a débuté sur deux rivières de BasseNormandie, l'Orne et la Touques. Les populations de ces deux cours d'eau ont été caractérisées à l'aide de paramètres biométriques et scalimétriques. Dans le cadre de ce travail, les écailles de plus de 10000 truites de mer adultes ont été analysées afin de mettre au point, voire de valider des critères d'interprétation de l'âge, des structures scalimétriques et des différentes phases biologiques de l'espèce. Les résultats de cette étude sont présentés ici.

\section{TECHNIQUES ET MÉTHODES}

Plus de 10.000 adultes de truite de mer ont été capturés principalement par piégeage de 1981 à 1988 sur l'Orne et la Touques, deux rivières de Basse-Normandie dont la situation géographique et les caractéristiques physiques ont déjà été présentées (RICHARD, 1981).

Trois à cinq écailles par individu ont été nettoyées dans une solution de soude à $10 \%$ et montées entre lame et lamelle.

Les définitions correspondant aux différentes structures observées sur l'écaille sont celles utilisées pour la mise au point des normes d'interprétation des écailles de saumon atlantique: circulus, bande hivernale et estivale, check, zone annuelle, annulus, reprise de croissance, érosion et marque de frai (ANONYME, 1984 et 1988).

Pour chaque poisson, un âge a été donné tenant compte des deux phases du cycle biologique (juvénile en eau douce et adulte en mer). L'âge marin a été noté de la manière conventionnelle suivante: $A+\operatorname{avec} A=$ nombre d'annuli présents sur la partie marine de l'écaille; le + signifie qu'ily a toujours chez la truite de mer une reprise de croissance après le dernier hiver marin. La présence d'une ou plusieurs marques de frai est notée à l'aide d'un exposant dont la valeur correspond au numéro de l'hiver, sur la partie marine de l'écaille, où a eu lieu la reproduction. Ainsi, l'âge d'une truite de mer ayant passé 2 ans en eau douce puis s'étant reproduite une fois après un séjour de 6 mois en mer s'écrit $2.1+$ '. L'avantage de cette formule par rapport à celle généralement utilisée (pour le même poisson l'âge serait noté 2.0+ 1MF (FAHY, 1978)) est de faire apparaître l'âge de mer total. Par ailleurs, lorsque des structures particulières, autres que la marque de frai, sont observées sur les écailles, elles ont été indiquées sous forme de sigles placés également en exposant. Ainsi une truite de mer âgée de $2.1+{ }^{2 R}$ signifie que le poisson a séjourné deux ans en eau douce et un an et demi en mer et que l'hiver situé sur la partie marine de l'écaille est constitué de deux bandes de stries fines resserrées.

L'âge marin à la première reproduction a été utilisé pour définir les types de truite de mer.

Dans de nombreux cas, l'interprétation des écailles a été validée par la recapture (ligne, filet, piège) d'individus marqués soit au stade smolt (marque carlin) soit à celui de "finnock" (1) (marque nouille).

Pour chaque figure présentée, la légende comprend dans l'ordre: le nom de la rivière, la date de capture du poisson, sa taille (longueur fourche en $\mathrm{mm}$ ), ses âges de rivière et de mer et les observations.

\section{RÉSULTATS}

\subsection{Age d'eau douce} rivière.

La quasi totalité $(98,5 \%)$ des truites de mer étudiées dévalent après 1 ou 2 hivers passés en

La phase juvénile en eau douce se caractérise généralement par des saisons de croissance régulières et bien définies, ce qui se traduit sur l'écaille par une nette alternance des bandes hivernales et estivales (fig. 1). Toutefois, on peut noter que:

(1) Finnock : terme écossais synonyme de post-smolt désignant les truites de mer retournant en rivière dès l'été suivant leur smoltification. 
- quels que soient l'âge d'eau douce et la rivière, le premier anneau hivernal ne se marque souvent que par un changement important de l'espacement des circuli entre les deux zones annuelles (fig. 2 et 3 ),

- une croissance plus ou moins continue jusqu'au stade smolt peut s'opposer à la localisation correcte du ou des hivers d'eau douce (fig. 4 et 5 ).

Il existe chez de nombreux individus une phase de transition sur l'écaille située entre les phases rivière et mer et correspondant à la période de dévalaison des juvéniles avant leur passage en mer (stade smolt) (validation par marquages). Elle est caractérisée par une reprise de croissance printanière, en moyenne d'autant plus forte que l'individu est plus jeune et/ou que la rivière est plus longue (fig. 6 et 7). A la fin de cette phase smolt peut apparaitre un check assez net (fig. 8).

\subsection{Age de mer}

\subsubsection{Type de truite de mer}

L'étude des écailles récoltées sur les deux rivières permet de classer les truites de mer en quatre types en fonction de la durée du séjour marin avant la première reproduction. Chaque type est illustré par une écaille de référence.

Type $0=3$ à 6 mois de mer ("Finnock"), (fig. 9).

Type $1=1$ hiver soit deux saisons de mer correspondant à $13-18$ mois de croissance marine. Les deux exemples donnés montrent une configuration sensiblement différentes de la bande hivernale marine entre les poissons des deux rivières (fig. 10 et 11).

Type $2=2$ hivers soit trois saisons de mer correspondant à 25-30 mois de croissance marine. Ce type, présent sur les deux rivières, se rencontre essentiellement sur l'Orne (fig. 12 et 13). Par ailleurs, il faut noter, dans quelques cas, une certaine difficulté pour délimiter précisément sur l'écaille la première bande hivernale (fig. 13 et 14 )

Type $3=3$ hivers soit quatre saisons de mer signifiant 35 à 40 mois de croissance marine. Ce type, rare, n'est rencontré que sur l'Orne (fig. 15).

\subsubsection{Marque de frai}

La marque de frai chez la truite de mer se définit par :

- la présence d'une ligne continue plus ou moins prononcée faisant le tour de l'écaille (fig. 16 et 17). L'érosion touche, d'abord et le plus fortement, la partie postérieure. Les côtés sont moins érodés et l'extrémité antérieure est généralement peu touchée (fig. 18).

- une configuration caractéristique des circuli entourant cette ligne. Lors de la reprise de croissance après le frai, les circuli sont déposés parallèiement à la ligne de résorption qui coupe les circuli formés précédemment. De plus, l'espacement et l'épaisseur des nouveaux circuli sont plus importants qu'avant la reproduction (fig. 19 et 20).

L'intensité de la marque de frai mesurée notamment d'après l'usure de la partie postérieure de l'écaille est croissante avec l'âge et la taille (fig. $21 a, b, c$ et d). Ceci peut aller jusqu'à empêcher la détermination correcte de l'âge de mer des gros individus à la première reproduction, en raison d'une importante érosion du champ antérieur de l'écaille (fig. 22).

La truite de mer est un poisson à frais multiples pouvant se reproduire sur ces deux rivières jusqu'à cinq à six fois (fig. 23 et 24).

Si la détection de la marque de reproduction est généralement aisée, certaines difficultés peuvent apparaître et sont liées à:

- une plus faible intensité de cette marque chez les poissons de type 0 (fig. 25). Toutefois, l'identification de la première marque de frai peut être facilitée par le fait que, sur un même poisson. les marques successives se forment de la même manière (fig. 26).

- la présence de pseudo-marques de frai sur le premier ou le second hiver post dévalaison. Ces structures se caractérisent par des pincements des circuli et une usure touchant les côtés de la bande hivernale tandis qu'aucune érosion ne touche la partie postérieure (fig. 27 et 28).

- l'âge élevé des poissons. Dans ce cas, il peut y avoir d'une part une résorption de la surface de l'écaille (fig. 29) et d'autre part un tassement voire un recouvrement des dernières marques de frai en bordure de l'écaille (fig. 30 ).

\subsubsection{Structures particulières}

L'examen des écailles collectées sur les deux cours d'eau a révélé l'existence de structures particulières qui se marquent sur la zone annuelle et le premier hiver du stade post-smolt. Ces structures, au nombre de trois, sont les suivantes:

\section{- bande hivernale à stries fines}

Le premier hiver post-dévalaison est représenté par un ensemble $(n>5)$ de circuli fins et resserrés apparaissant brutalement après la bande estivale marine (fig. 31 a et b). Cette structure est 
bien apparente dans la partie antérieure de l'écaille mais se resserre et disparaît sur les côtés. Ces circuli ont une épaisseur et un écartement analogues à ceux produits pendant la phase de croissance juvénile en eau douce. Cette structure s'accompagne d'une absence d'érosion.

Ces diverses constatations laissent penser que la truite a passé son hiver post-dévalaison en rivière sans se reproduire avec une poursuite ralentie de la croissance de l'écaille (fig. 32a et b)

\section{- bande hivernale réduite}

Le premier hiver post-dévalaison se présente sous forme d'un simple resserrement faisant le tour de l'écaille et ne comportant qu'un nombre réduit de circuli $(n<5)$ (fig. 33a). On note fréquemment la présence d'une faible érosion visible essentiellement sur les côtés du resserrement hivernal (fig. 33b). Comme dans le cas précédent, il s'agirait d'un hivernage en rivière sans reproduction mais sans croissance de l'écaille (fig. $34 a$ et b).

\section{- double resserrement. Cette structure regroupe deux cas:}

- deux resserrements identiques séparés par une zone de forte croissance apparaissent sur l'écaille (fig. 35a et b). Souvent on observe une usure latérale, parfois prononcée, au niveau du premier resserrement (fig. 36). Cette structure correspond alors à une incursion estivale en eau douce au stade finnock immature; l'érosion observée serait due à un changement brutal de milieu s'accompagnant de profondes modifications de l'activité alimentaire. Ensuite, le poisson repart grossir en mer avant de refaire, en fin d'hiver, une nouvelle incursion (second resserrement) dans la partie aval ou l'estuaire du cours d'eau (fig. 37a, b et c).

- deux resserrements différents se succèdent sur l'écaille (fig. 38a et b). Après le premier, identique au cas évoqué précédemment, apparaît une bande hivernale marine (deuxième resserrement). Ainsi, après une incursion estivale en rivière, le poisson redescend rapidement en mer pour y poursuivre sa croissance.

\section{DISCUSSION}

Les résultats de ce travail permettent de dégager plusieurs points importants quant à la description et l'interprétation des écailles de truites de mer adulte de l'Orne et de la Touques.

\section{La distinction entre trois phases de croissance de l'écaille.}

En effet, la présence des zones rivière, transition et mer apparaît caractéristique d'un salmonidé migrateur anadrome à nos latitudes (BAGLINIËRE, 1985). Leur succession sur un grand axe de l'écaille se traduit par une augmentation de l'épaisseur et de l'espacement des circuli.

\section{Une bonne caractérisation de la phase juvénile en eau douce.}

L'interprétation de la phase en eau douce des écailles de truite de mer de l'Orne et de la Touques présente en règle générale peu de difficultés compte tenu du fort taux de croissance en rivière de ces populations. Les deux difficultés d'interprétation qui subsistent sont par contre lièes à la valeur élevée de ce paramètre:

(1) croissance continue ne permettant pas de localiser correctement les lignes d'arrêt de croissance

(2) confusion possible du dernier hiver d'eau douce avec le premier hiver marin chez les individus montrant une forte reprise de croissance printanière au début de la deuxième année d'eau douce.

Durant cette phase juvénile en eau douce, il n'a pas été observé de marques de frai dont la structure sur l'écaille a été par contre constatée et définie chez le jeune saumon au même stade (BAGLINIERE et MAISSE, 1985) et chez la truite de rivière (MAISSE et al., 1987).

\section{La définition de quatre types de truite de mer}

La présence de ces différents types a été confirmée sur d'autres bassins français depuis celui de l'Adour jusqu'aux fleuves picards. Cette définition constitue, dans le cas de suivis de population pluriannuels, un outil précieux pour caractériser et comparer les stocks. Ainsi dans le cadre de cette étude, il apparaît une nette différence entre les truites de mer de l'Orne à long séjour marin et celles de la Touques à retour plus précoce (RICHARD, 1986, EUZENAT et al., 1990).

\section{La définition de la marque de frai après un premier séjour marin}

Les critères retenus pour caractériser la marque de reproduction sont en accord avec ceux proposés par BACKIEL et SYCH (1958) pour des populations de truites de mer de taille assez proche de celles étudiées. Cependant, dans le cas de poissons de petite taille, l'identification de cette structure sur l'écaille apparaît beaucoup plus difficile en raison d'un phénomène de résorption de l'écaille moins prononcé, pouvant aller jusqu'à l'absence de frai au regard des critères habituels. Néanmoins, l'utilisation de ces critères doit permettre généralement de ne pas confondre cette structure avec celle de pseudo-marques de frai.

La comparaison de cette marque de reproduction chez le saumon et la truite de mer amène deux remarques principales: 
- la localisation de l'érosion diffère selon l'espèce, elle concerne surtout la partie postérieure de l'écaille chez la Truite de mer, les champs latéraux et le bord antérieur chez le Saumon (BAGLINIERE, 1985). II s'agit là d'un critère permettant de distinguer les deux espèces même si l'érosion du bord postérieur de l'écaille apparaît également importante chez les saumons mâles adultes (BAGLINIERE, 1985).

- la forte intensité de la marque de frai chez le saumon traduit bien le fait que cette espèce se reproduit exceptionnellement plus d'une fois (en moyenne 1,1\% dans les stocks: BAGLINIERE et PORCHER (données non publiées)). De plus, cette forte érosion rend souvent difficile voir impossible la détermination de l'âge de mer de première remontée. Inversement, la plus faible intensité de cette marque chez la truite de mer est caractéristique d'une espèce à frai multiple et ne gêne généralement pas la détermination de l'âge de mer.

\section{dévalaison.}

La presence de structures particulières en liaison avec les différents comportements post-

La phase de croissance de l'écaille correspondant au stade post-smolt (depuis le passage en mer jusqu'à la fin de l'hiver suivant) demeure encore la zone la plus délicate à interpréter. En effet, une analyse superficielle de ces structures amène à surestimer l'âge marin du poisson soit en les confondant avec des marques de frai de faible intensité (LE CREN, 1985) soit en comptabilisant à tort des structures dédoublées (voir le double resserrement post-dévalaison caractéristique des poissons de la Touques).

Dans ie cas présent, l'interprétation de ces structures n'a pu être validée qu'à l'aide de poissons recapturés-marqués ou de sujets contrôlés périodiquement durant cette phase.

Ainsi l'existence et la confirmation de ces structures montre la complexité des comportements post-dévalaison de la truite de mer, quelle que soit la population étudiée, c'est-à-dire indépendamment de la durée du séjour marin.

\section{CONCLUSION}

Cette étude sur la description et l'interprétation des écailles de Truite de mer de l'Orne et de la Touques a permis:

- d'apporter des compléments indispensables à la caractérisation biométrique des stocks et de les comparer à l'aide des types définis dans une première approche,

- de constituer des critères de référence scalimétriques qui pourront servir de base pour homogénéiser les normes de lectures au niveau national. Ce travail a déjà commencé au sein du groupe salmonidés migrateurs du Groupe Permanent de Concertation des Poissons Amphihalins. Cependant, compte tenu de la complexité du comportement post-dévalaison de la truite de mer, il parait indispensable de mettre en place des procédés de validation de la lecture d'écaille lors de l'étude de nouveaux stocks,

- de fournir des éléments utilisables dans le domaine de la gestion. Ceci s'est concrétisé par la mise en place en Basse-Normandie d'une réglementation de pêche mieux adaptée aux stocks.

\section{BIBLIOGRAPHIE}

ALM G., 1950. The sea trout population in the Ava stream. Rep. Inst. Freshwat. Res., Drottningholm, $31: 26-51$.

ANONYME, 1984. Atlantic Salmon scale reading. Report of Atlantic Salmon scale reading workshop, Aberdeen, Scotland, 23-28 april 1984, ICES, 1 vol., $17 \mathrm{p}$.

ANONYME, 1988. Atlantic Salmon scale reading. Report of the second Atlantic Salmon scale reading workshop, Aberdeen, Scotland, 12-14 october 1988, ICES, 1 vol., 16 p.

BACKIEL T., SYCH R., 1958. Scales resorption and spawning marks in sea trout (Salmo trutta L.) from polish waters. Roc. Nauk. Rol., 73 ser. B : 119-148.

BAGLINIERE J.L., 1985. La détermination de l'âge par scalimétrie chez le saumon atlantique dans son aire de répartition méridionale : utilisation pratique et difficultés de la méthode. Bull. Fr. Pêche Piscic., 298 : 69-105.

BAGLINIERE J.L., LE LOUARN H., 1987. Caractéristiques scalimétriques des principales espèces de poisson d'eau douce en France. Bull. Fr. Pêche Piscic., 306: 1-39.

BAGLINIERE J.L., MAISSE G., 1985. Precocious maturation and smoltification in wild atlantic salmon in the armoricain Massif, France. Aquaculture, $45:(1-4): 249-263$.

BEALL E., DAVAINE P., 1988. Analyse scalimétrique de la truite de mer (Salmo trutta L.) : formation des anneaux et critères d'identification chez les individus sédentaires et migrateurs d'une même population acclimatée aux lles Kerguelen (TAAF). Aquat. Living Resour., 1 : 3-16. 
CHELKOWSKI Z., 1969. The sea trout, Salmo trutta $m$. trutta L., of the Pomeranian coastal rivers. Prz. Zool., 13 (1) : 72-91.

CHRZAN F. 1962. The scales of sea trout of known age. ICES Salmon and trout Committee, CM $1962: 25,3 \mathrm{pp}$.

DAHL R., 1909. The assessment of age and growth in fish. Int. Revue der gesamt. Hydrobiol. und Hydrogr., $2: 758-769$.

EUZENAT G., FOURNEL F., RICHARD A., 1990. La truite de mer (Salmo trutta L.) en Normandie/ Picardie. In "Biologie et écologie de la Truite (Salmo trutta L.) en France". Ed., J.L. BAGLINIERE et G. MAISSE, I.N.R.A., Paris (sous presse).

FAHY E., 1978. Variation in some biological characteristics of Bristish sea trout, Salmo trutta $L$. J. Fish Biol., $13:$ 123-138.

JARVI T.H., MENZIES N.J.M., 1936. The interpretation of the zones on scales of salmon, sea trout and brown trout. Cons. Perm. Expl. Mer XCVII, 1 vol., $63 \mathrm{p}$.

JENSEN K.W., 1968. Sea trout (Salmo trutta L.) of the river Istra, Western Norway. Rep. Inst. Freshwat. Res., Drottningholm, $48: 187-213$.

JONSSON B., 1985. Life history patterns of freshwatter resident and sea-run migrant brown trout in Norway. Trans. Am. Fish. Soc., $114: 182-194$.

LE CREN E.D., 1985. The biology of the sea trout. Symposium Oct. 84, Atlantic Salmon Trust, $42 \mathrm{p}$.

MAISSE G., BAGLINIERE J.L. et LE BAIL P.Y., 1987. Dynamique de population de truite commune (Salmo trutta) d'un ruisseau breton (France): les géniteurs sédentaires. Hydrobiologia, $148: 123-130$.

PRATTEN D.J., SHEARER W.M., 1983. Sea trout of the North Esk. Fish. Mgmt., $14: 49-65$.

RICHARD A., 1981. Observations préliminaires sur les populations de truite de mer (Salmo trutta L.) en Basse-Normandie. Bull. Fr. Piscic., 283 : 114-124.

RICHARD A., 1986. Les populations de Truite de mer (Salmo trutta L.) des rivières Orne et Touques (Basse-Normandie); Scalimétrie; Sexage; Caractéristiques biométriques et démographiques. Thèse $3^{\ominus}$ cycle. Fac. Sci. Univ. Rennes, 1 vol., $54 \mathrm{p}$.

ROULE L., 1920. Etude sur le saumon des eaux douces de la France considéré du point de vue de son état naturel et du repeuplement de nos rivières. Minist. Agriculture, Paris, 1 vol., $178 \mathrm{p}$.

SYCH R., 1967a. Confidence estimation of a fish age determination from scales as exemplified by sea-trout (Salmo trutta L.) Roczn. Nauk Roln., H90 (2) : 281-303.

SYCH R. 1967b. Interpretation of the scales of Vistula sea-trout (Salmo trutta L.) Acta Hydrobiol., $9(3-4)$ : 231-280.

VIBERT R., 1950. Recherches sur le saumon de l'Adour (Salmo salar L.) (Ages, croissance, cycle génétique, races), 1942-1948. Ann. St. Cent. Hydro. appl., $3:$ 27-148.

ZARNECKI S., 1960. General conclusions on the scale-reading of salmon, sea trout and brown trout originating in the Vistula. ICES ANACAT, Fish. Comm., $128: 1-3$.

ZARNECKI S., 1973. Differentiation of Atlantic salmon (Salmo salar L.) and of sea trout (Salmo trutta L.) from the Wisla (Vistula) river into seasonal populations. Acta Hydrobiol., 5 (2/3) : 255-294. 


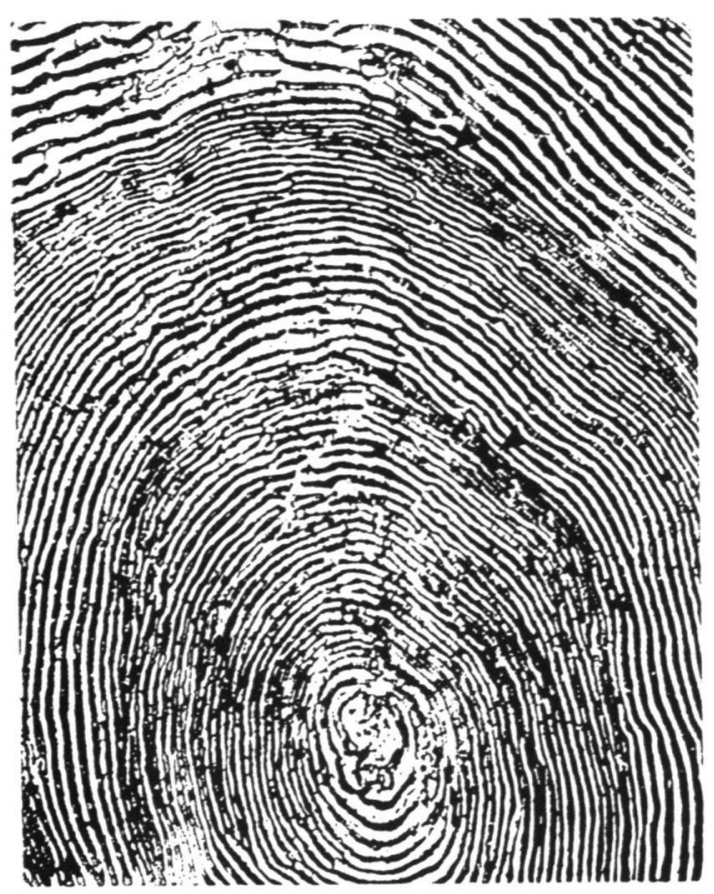

Fig. 1 : Touques, 28.7.83, 406, 2.0+, bonne alternance des bandes hivernales $(4)$ et estivales en eau douce.

Fig. 1 : Touques, 28.7.83, 406, 2.0+, winter and summer bands very well separated in freshwater $(1)$

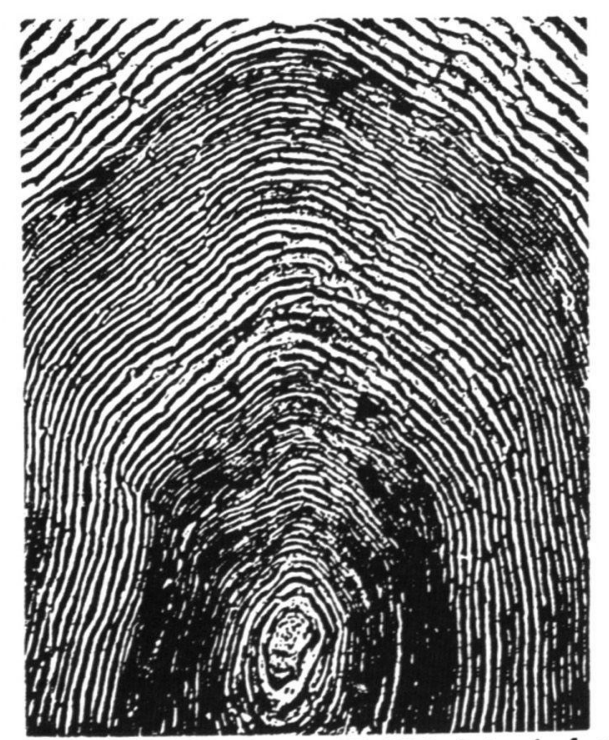

Fig. 3 : Touques, 9.6.87, 560, 2.1+, la forte reprise de croissance en deuxième année d'eau douce fait apparaître la fin du premier hiver $(\checkmark)$ ). On notera également que le pourtour de la phase eau douce est bien délimitée.

Fig. 3 : Touques, 9.6.87, 560, 2.1+, the high growth in the second freshwater year do appear the end of the first winter band, $(4)$. River zone is well delimited.

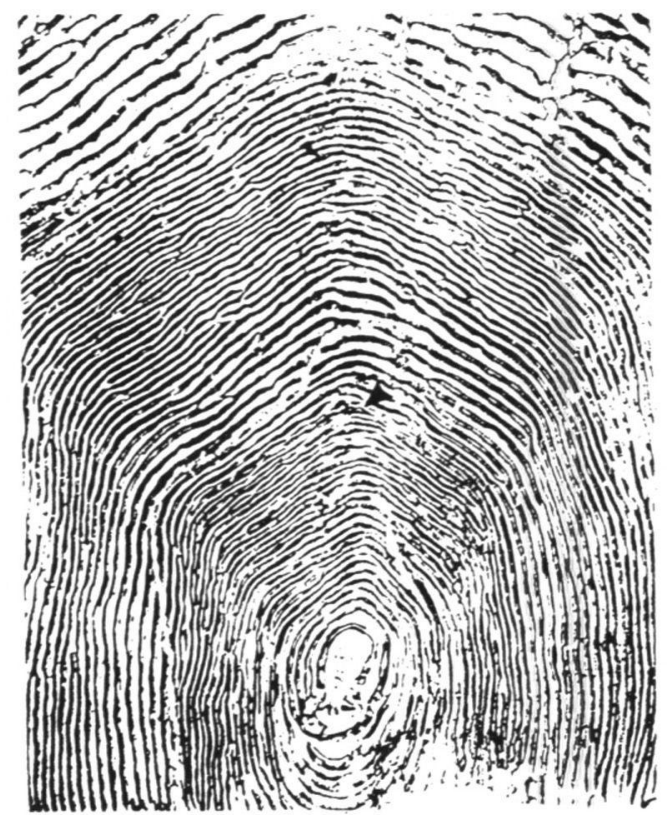

Fig. 2 : Orne, 16.6.87, 608, 2.2t, la forte reprise de croissance en deuxième année d'eau douce fait apparaître la fin du premier hiver $(4)$

Fig. 2 : Orne, 16.6.87, 608, 2.2+, the high growth in the second freshwter year do appear the end of the first winter band, ( 4 )

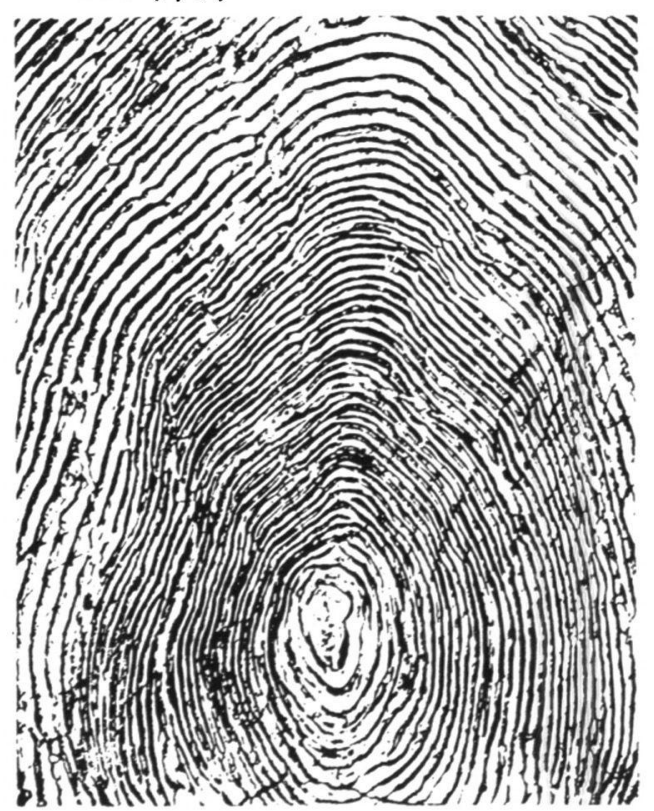

Fig. 4 : Orne, $30.11 .83,578,1$ probable. 1+, croissance continue en eau douce ne permettant pas de déterminer correc. tement la position du premier hiver d'eau douce.

Fig. 4 : Orne, 30.11.83, 578, 1 probably. 1+, the continuous growth in freshwater phase does not allow to determine the position of the first winter band. 


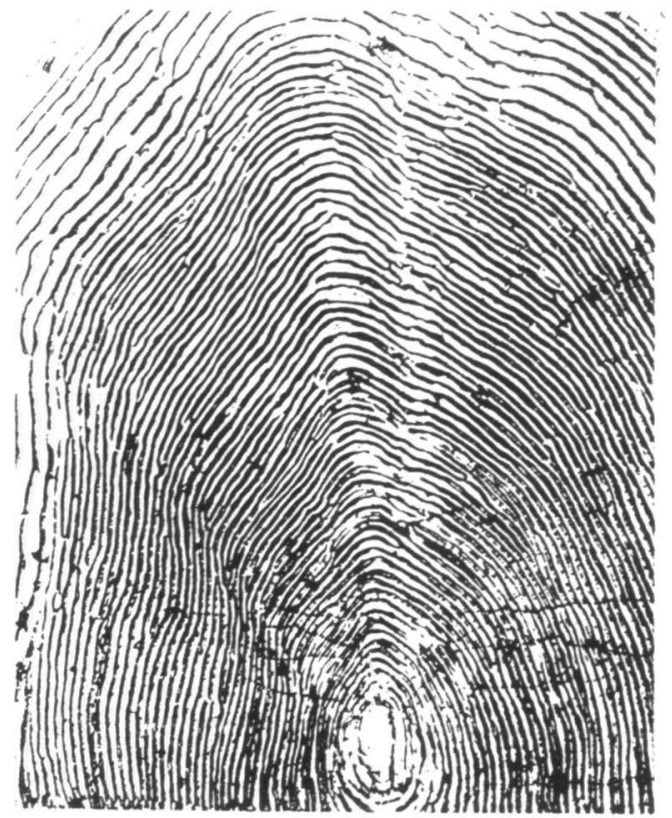

Fig. 5 : Touques, $7.7 .87,345,2$ probable. $0+$, croissance continue en eau douce ne permettant pas de localiser correctement les bandes hivernales.

Fig. 5 : Touques, 7.7.87, 345, 2 probably. $0+$, the continuous growth in freshwater phase does not allow correctly to determine the position of winter bands.

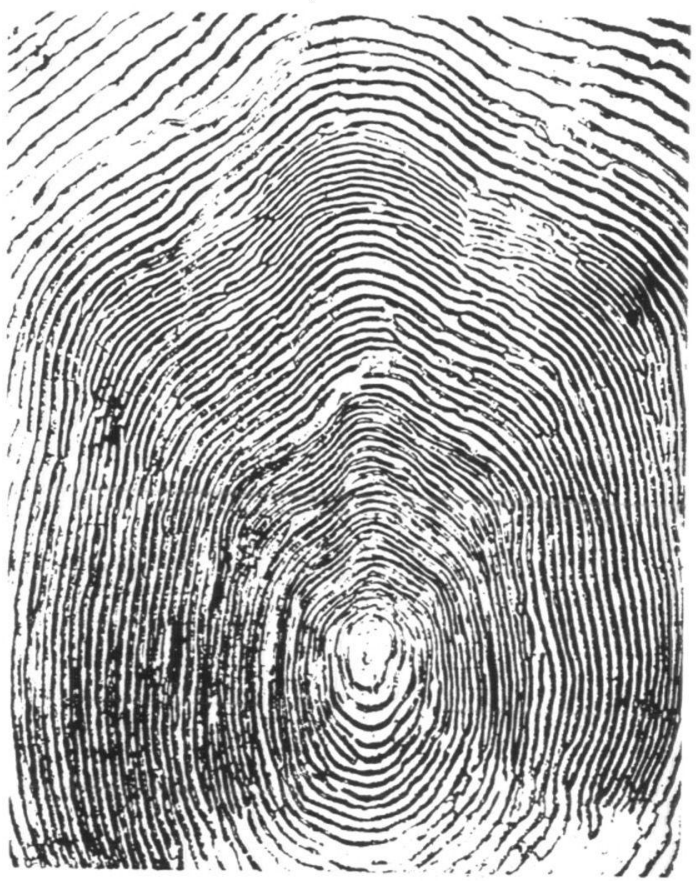

Fig. 7 : Touques, $7.7 .87,350,2+.0+$, phase de transition (croissance smolt) bien in. dividualisee mais moins importante que dans le cas précédent.

Fig. 7 : Touques, $7.7 .87,350,2+.0_{+}$, run-out phase well visible but less important as in figure 6 .

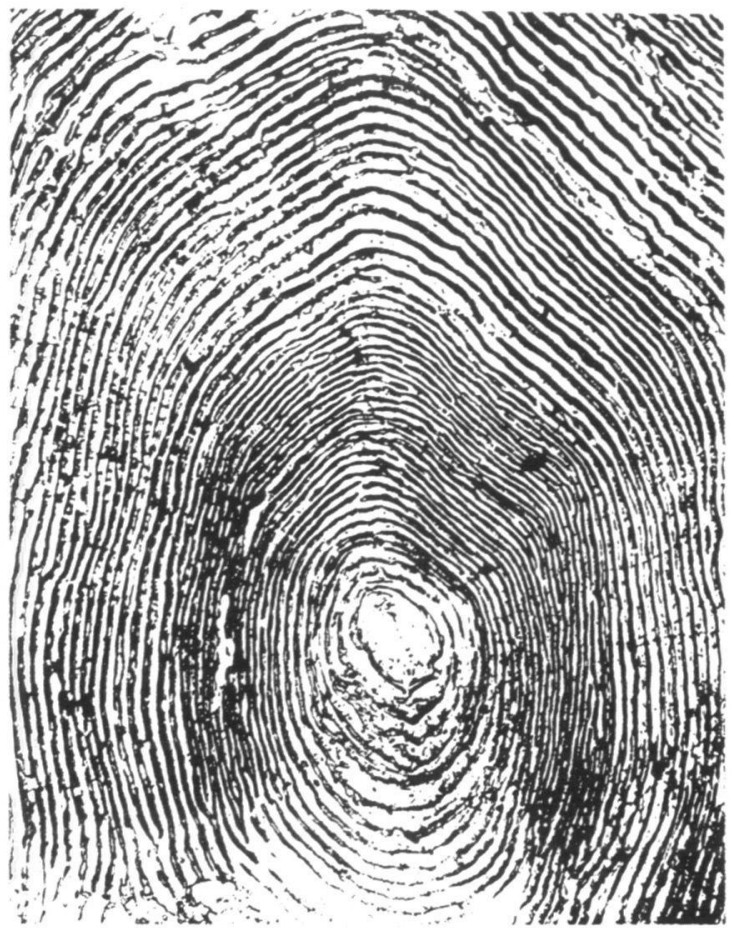

Fig. 6 : Orne, $23.10 .81,545,1+.1+$, phase de transition (croissance smolt) bien individualisée.

Fig. 6 : Orne, 23.10.81, 545, 1+.1+, run-out phase (smolt stage) well visible.

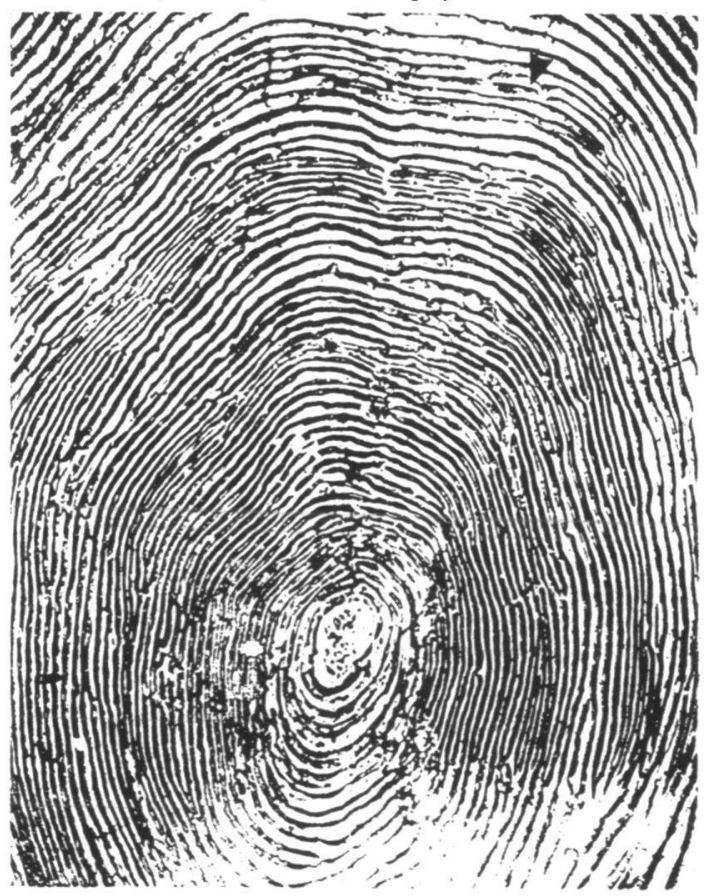

Fig. 8 : Touques, 22.7.83,370,2+.0+, présence d'un check $(4)$ en tin de phase de transition.

Fig. 8 : Touques, 22.7.83,370,2+.0+, presence of a check in the end of run-out phase (4). 


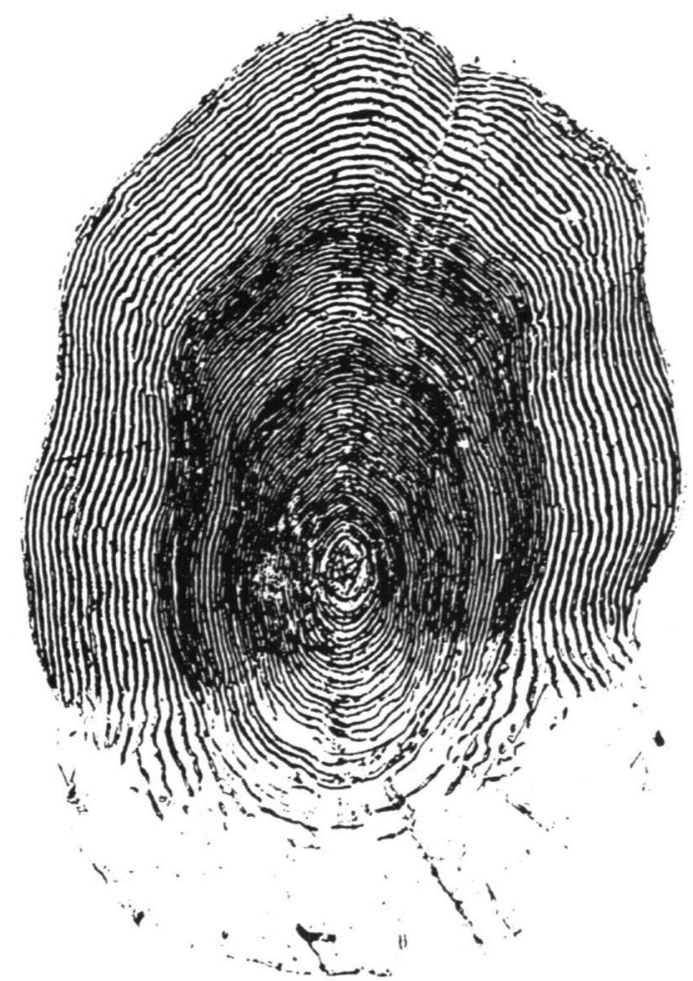

Fig. 9 : Touques, 10.8.83, 380, 2+.0+, type 0 ou finnock.

Fig. 9 : Touques, $10.8 .83,380,2+.0+$, type 0 or finnock.

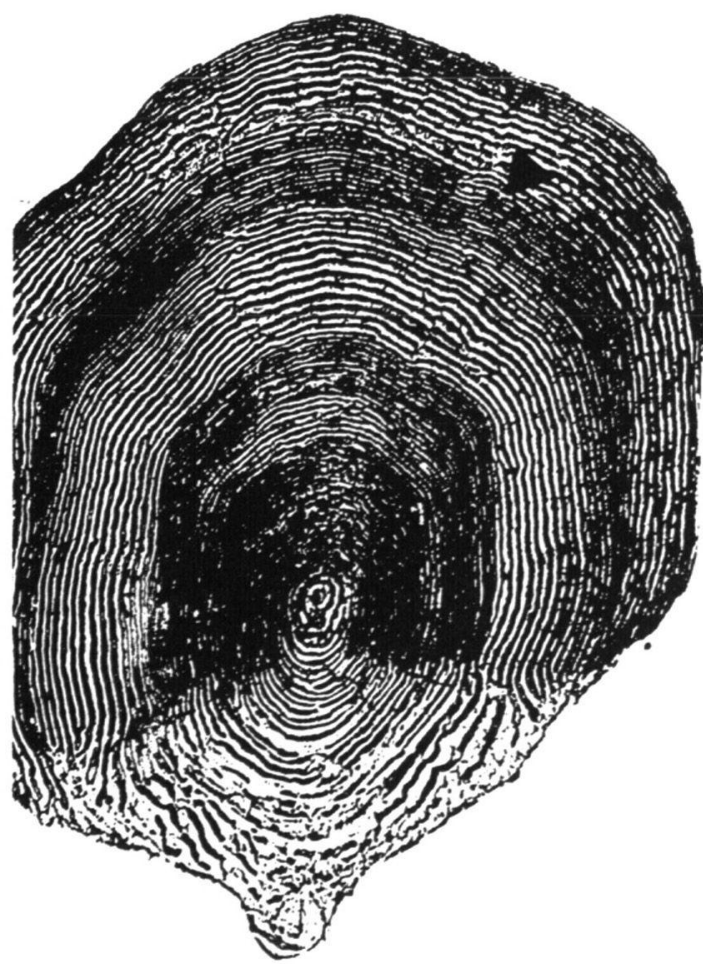

Fig. 11 : Touques, 22.6.84, 496, 2.1+, type 1 hiver marin (4).

Fig. 11 : Touques, 22.6.84, 496, 2.1+, type 1, sea winter band (1).

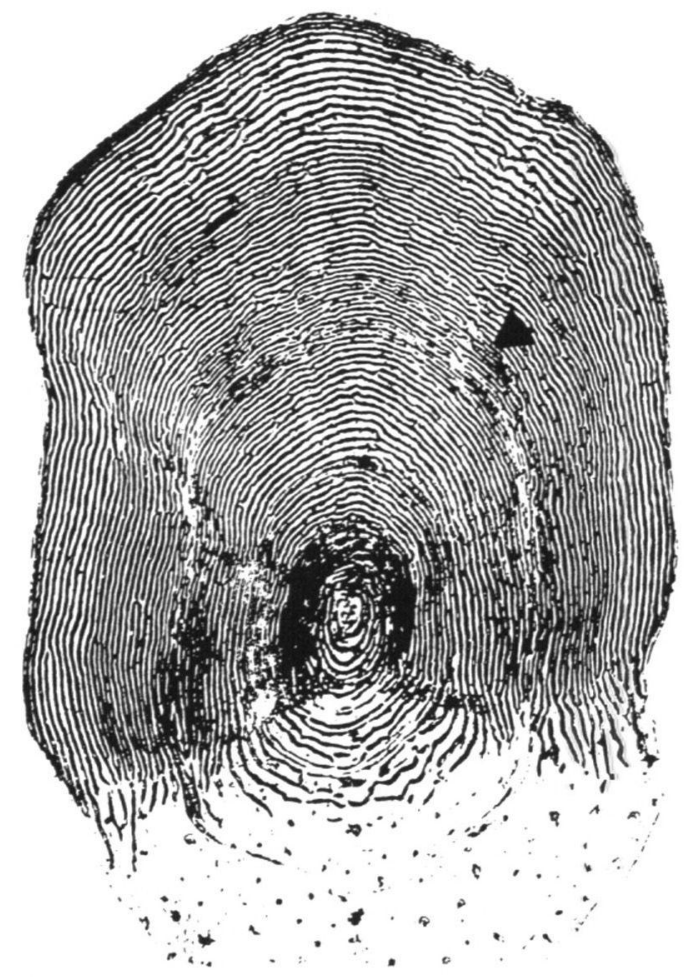

Fig. 10 : Orne, 3.6.87, 450, 1.1.t, type 1 hiver marin (4).

Fig. 10 : Orne, 3.6.87, 450, 1.1.t, type 1, sea winter band $(1)$.

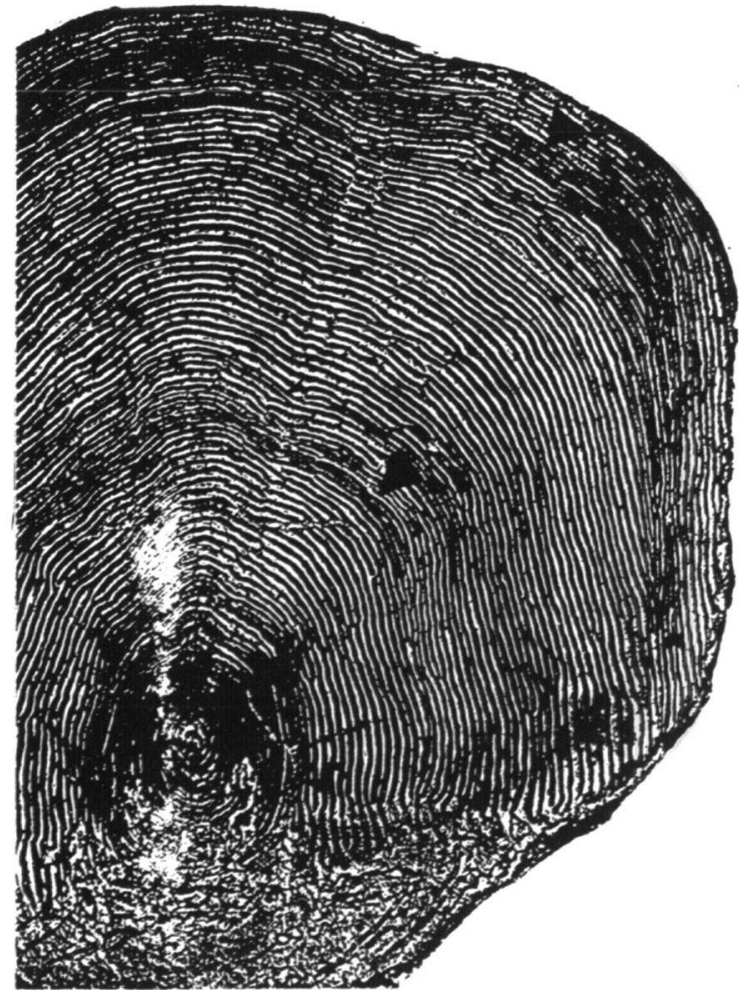

Fig. 12 : Orne, 15.12.83, 760, 1.2+, type 2, hiver marin ( 1 ).

Fig. 12 : Orne, 15.12.83, 760, 1.2+, type 2, sea winter band ( ). 


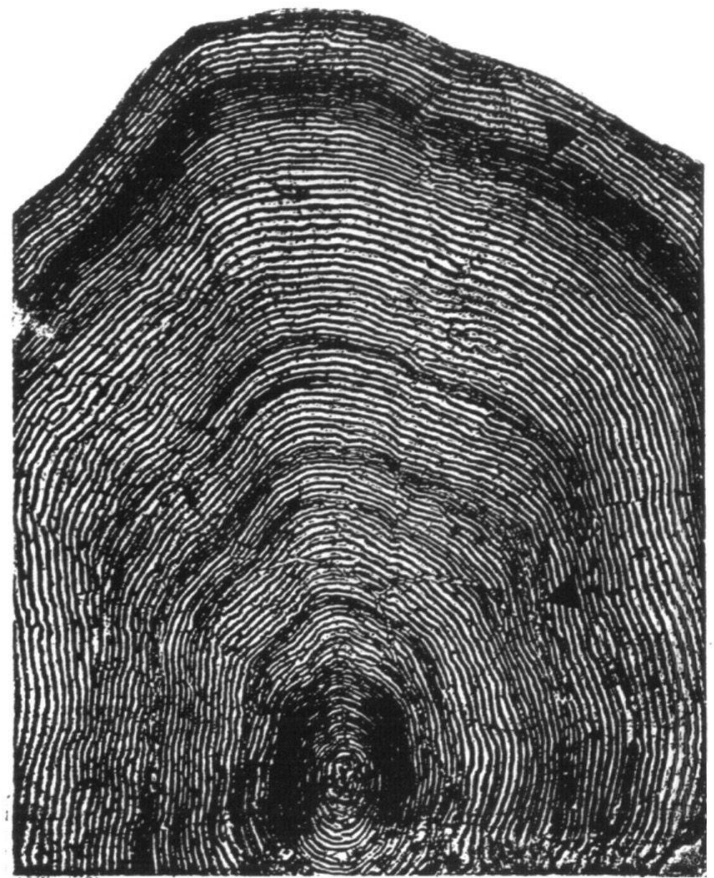

Fig. 13 : Orne, 29.5.88, 814, 1.2+, type 2 ; localisation difficile du premier hiver marin ; hiver marin (4).

Fig. 13 : Orne, 29.5.88, 814, 1.2+, type 2 ; difficult localization of the first sea winter band ; winter band $(4)$ ).

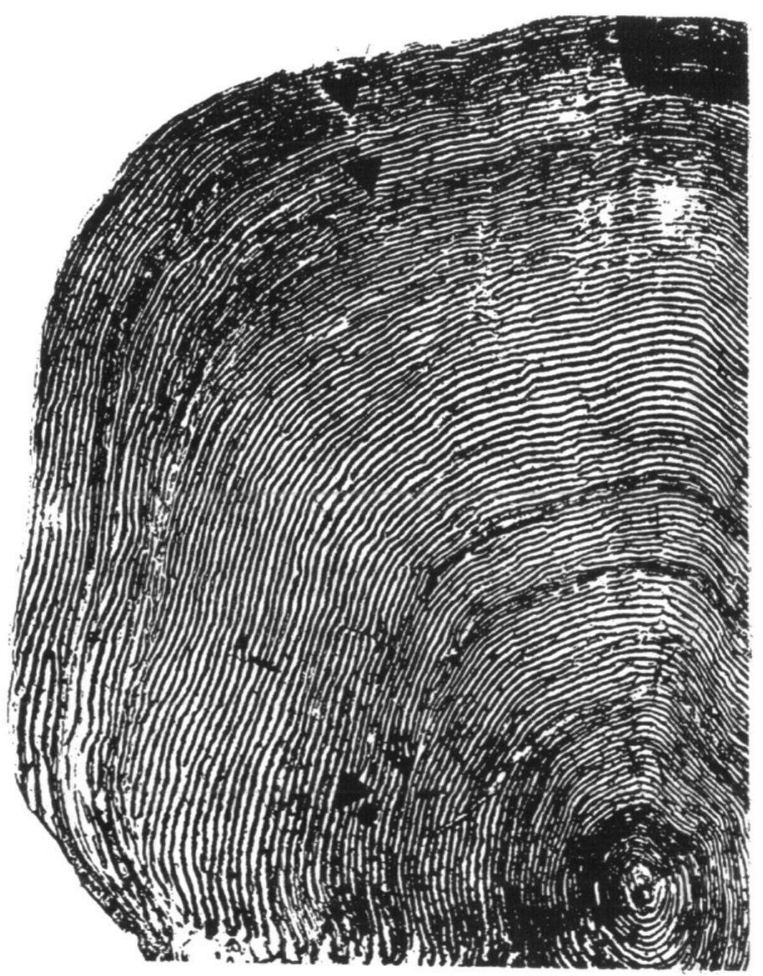

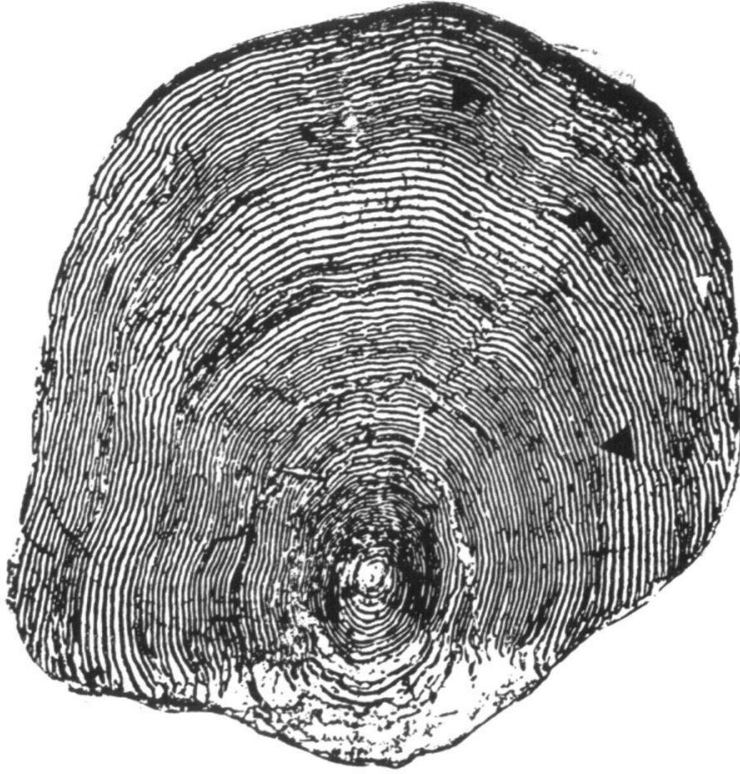

Fig. 14 : Touques, 12.9.81, 800, 1.2+, type 2 localisation difficile du premier hiver marin ; hiver marin (4).

Fig. 14 : Touques, $12.9 .81,800,1.2+$, type 2 difficult localization of the first sea winter band ; winter band $(\Lambda)$.

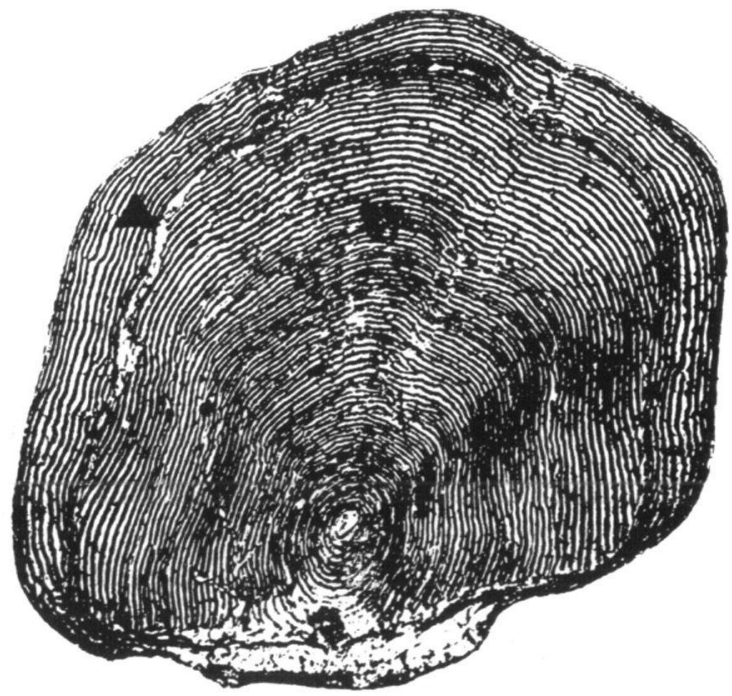

Fig. 16 : Orne, 4.7.83, 633, $1.2+^{2}$, présence d'une marque de frai typique ( 4 ) après deux saisons de croissance en mer.

Fig. 16 : Orne, $4.7 .83,633,1.2+^{2}$, presence of a typical spawning mark ( ) after two sea growth seasons.

Fig. 15 : Orne, 29.5.83, 847, 1.3+, type 3. II faut noter comme les cas précédents la difficulté pour positionner le premier hiver marin ( fig. 13 et 14); hiver marin ( ).

Fig. 15 : Orne, 29.5.83, 847, 1.3+, type 3. Difficult localization of the first sea winter band like in the figures 13 and 14 ; winter band $(A)$. 


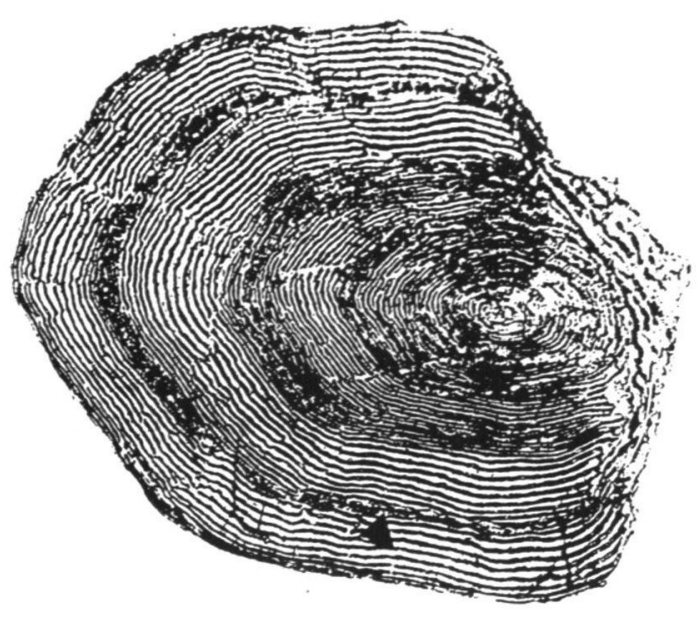

Fig. 17 : Touques, 23.7.84, 469, 2.1+, présence d'une marque de frai typique (4) après une saison de croissance marine.

Fig. 17 : Touques, 23.7.84, 469, 2.1+, presence of a typical spawning mark (4) after one sea growth season.

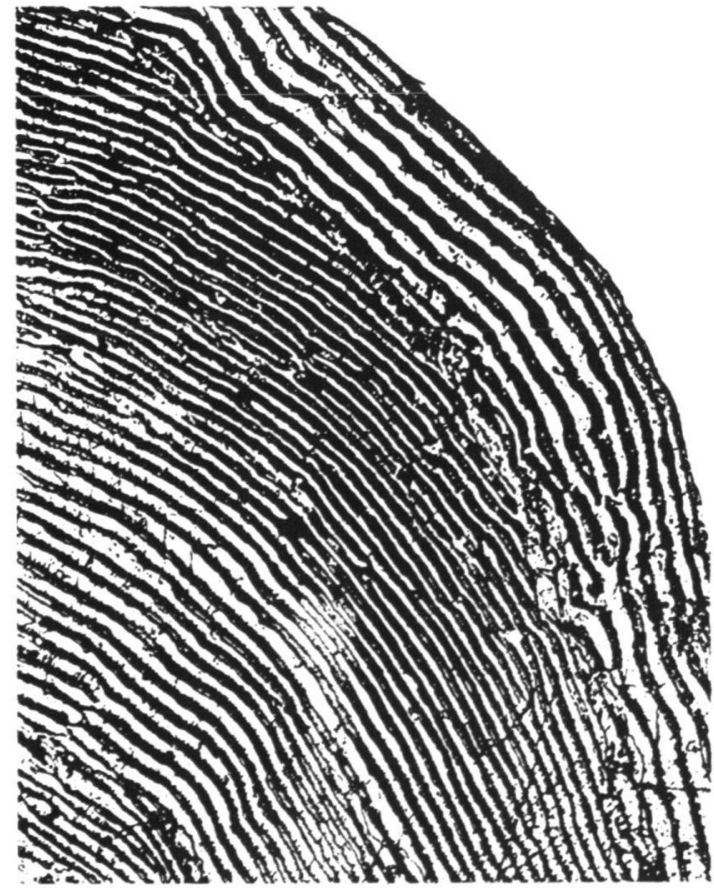

Fig. 19 : Orne, $4.7 .83,710,1.3+^{2}$, caractéristiques d'une marque de frai lors de la première reproduction.

Fig. 19 : Orne, $4.7 .83,710,1.3+^{2}$, characteristics of spawning mark during the first reproduction.

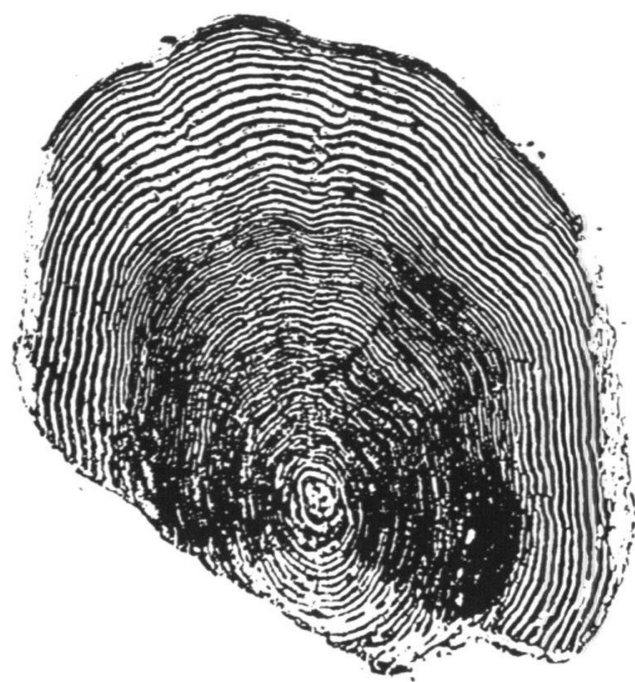

Fig. 18 : Touques, 23.10.84, 416, 2.0+, marque de frai en formation sur le bord antérieur et les champs latéraux de l'écaille.

Fig. 18 : Touques, 23.10.84, 416, 2.0+, beginning of the spawning mark on the anterior edge and the sides of the scale.

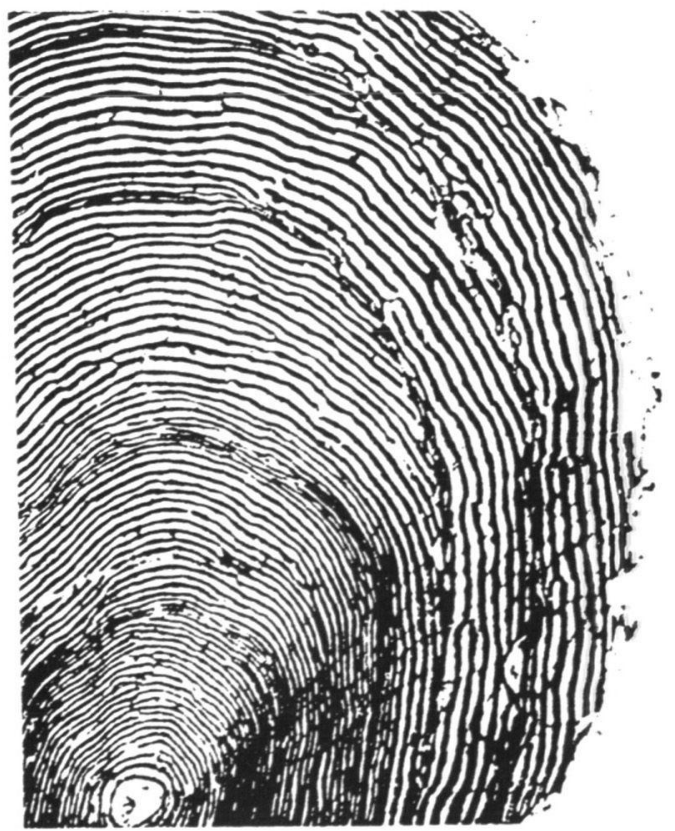

Fig. 20 : Touques, $19.11 .81,585,2.3^{1,2.3}$, caractéristiques des marques de reproduction chez un poisson ayant frayé au moins 2 fois.

Fig. 20 : Touques, $19.11 .81,585,2.3^{1,2,3}$, characteristics of spawning mark in a sea-trout having spawned two times already. 


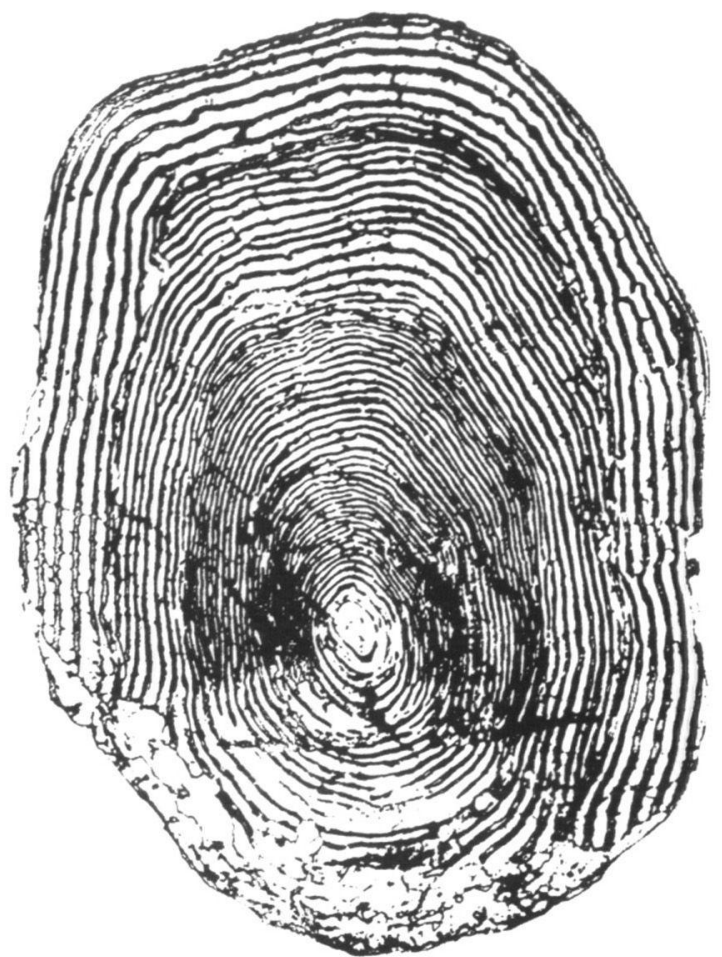

Fig. 21a : Touques, $19.9 .81,422,2.1+{ }^{1}$, reproduction au stade finnock.

Fig. 21a : Touques, 19.9.81, 422, $2.1+^{1}$, reproduction at Finnock stage.

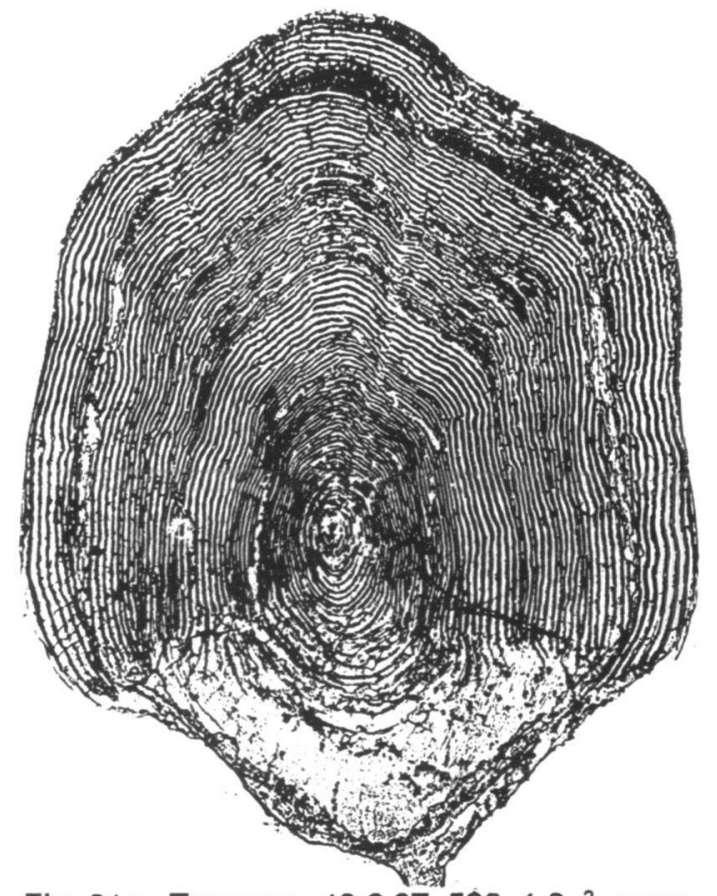

Fig. 21c : Touques, $12.8 .87,538,1.2+^{2}$, reproduction à l'âge $1+$ de mer (type 1 ).

Fig. $21 \mathrm{c}$ : Touques, $12.8 .87,538,1.2 t^{2}$, reproduction at $1+$ sea age (type 1 ).

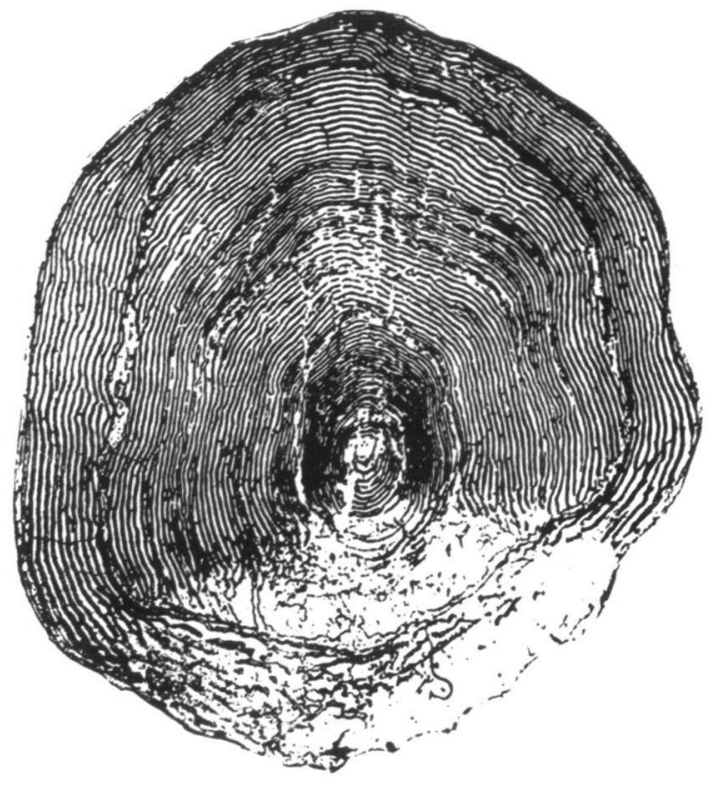

Fig. 21 b : Orne, 5.7.83, 665, $1.2+^{2}$, reproduction à l'áge 1+ de mer (type 1).

Fig. 21 b : Orne, 5.7.83, 665, $1.2+^{2}$, reproduction at $1+$ sea age (type 1).

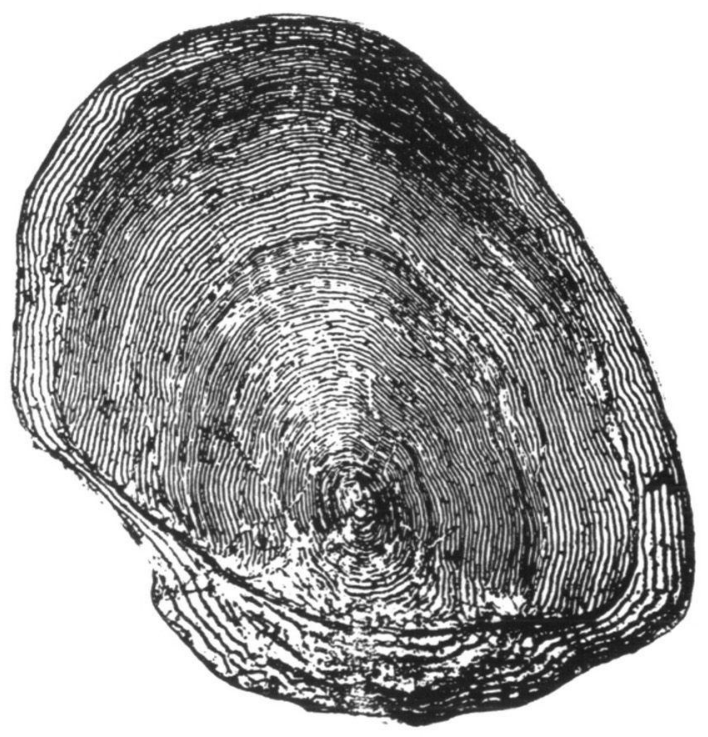

Fig. $21 \mathrm{~d}$ : Orne, $4.7 .83,710,1.3+^{3}$, reproduction à l'âge 2+ de mer (type 2).

Fig. $21 \mathrm{~d}$ : Orne, $4.7 .83,710,1.3+^{3}$, reproduction at $2+$ sea age (type 2).

Fig. 21 : Evolution de la marque de frai avec l'áge et la taille du poisson

Fig. 21 : Evolution of the spawning mark in relation to the age and size of fish. 


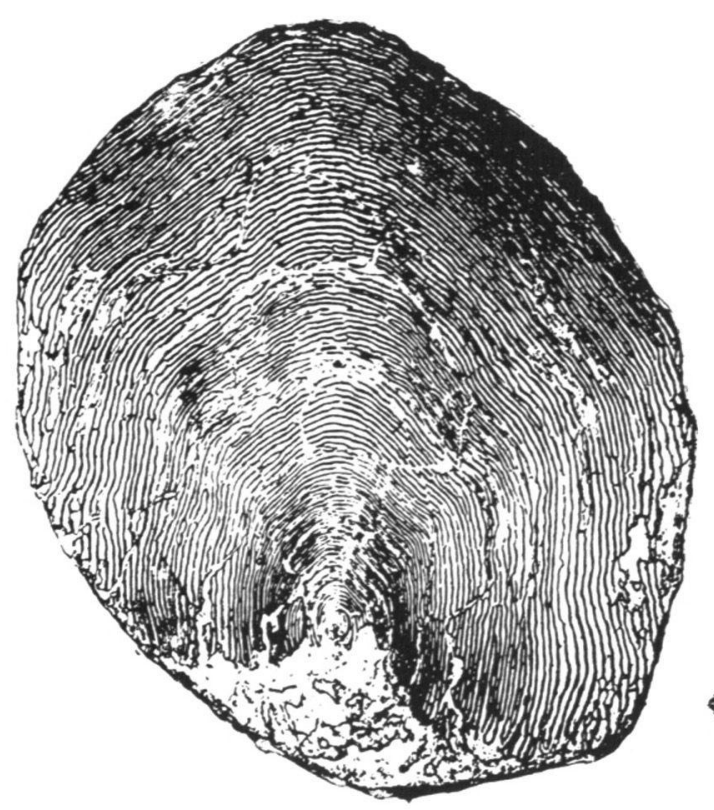

Fig. 22 : Orne, 19.10.83, 692, 1.2+ probable, érosion importante du champ antérieur de l'écaille ne permettant pas de déterminer avec précision l'âge de mer.

Fig. 22 : Orne, 19.10.83, 692, 1.2+ probably, the large erosion of the scale edge does not allow correctly to determine the sea age of the fish.

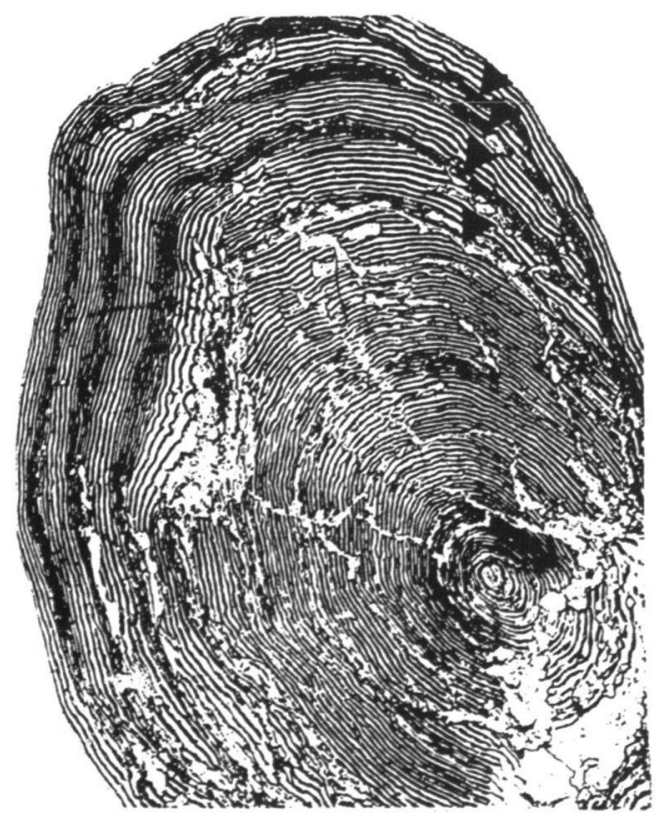

Fig. 24 : Orne, $6.6 .87,765,1.5+{ }^{2345}$, poisson s'étant reproduit 4 fois $(4)$, la première reproduction ayant eu lieu à l'áge 1+ de mer.

Fig. 24 : Orne, $6.6 .87,765,1.5 t^{2345}$, sea trout with 4 spawning marks $(4)$, the first reproduction occured after two sea growth seasons (1+ sea age).

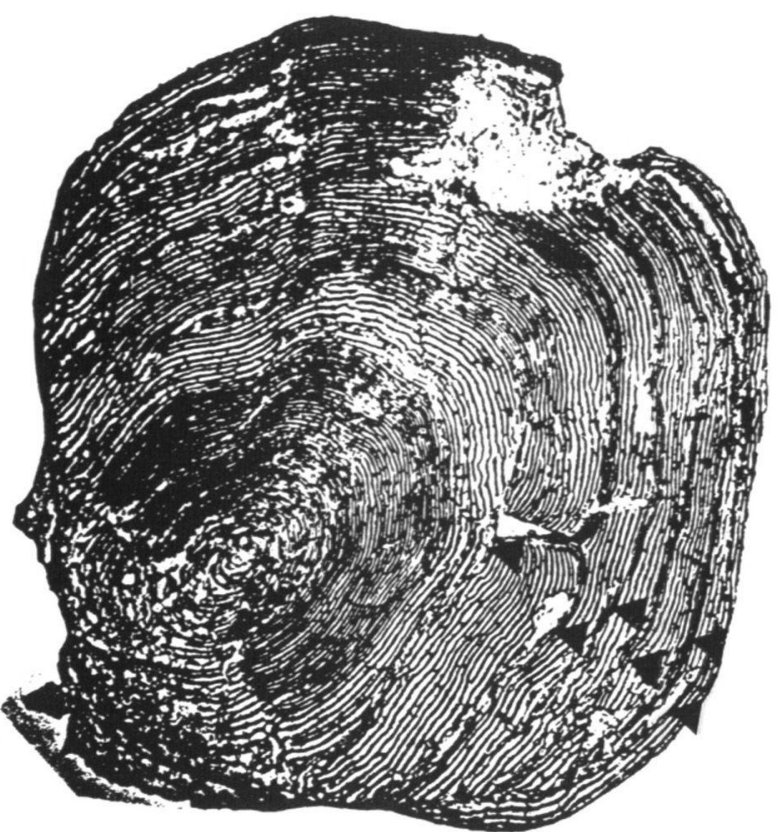

Fig. 23 : Touques, 24.6.85, 730, 2.6+ ${ }^{123456}$, poisson s'étant reproduit 6 fois $(4)$, la première reproduction ayant eu lieu au stade finnock.

Fig. 23 : Touques, $24.6 .85,730,2.6+{ }^{123456}$, sea trout with six spawning marks $(4)$, the first reproduction occured at finnock stage.

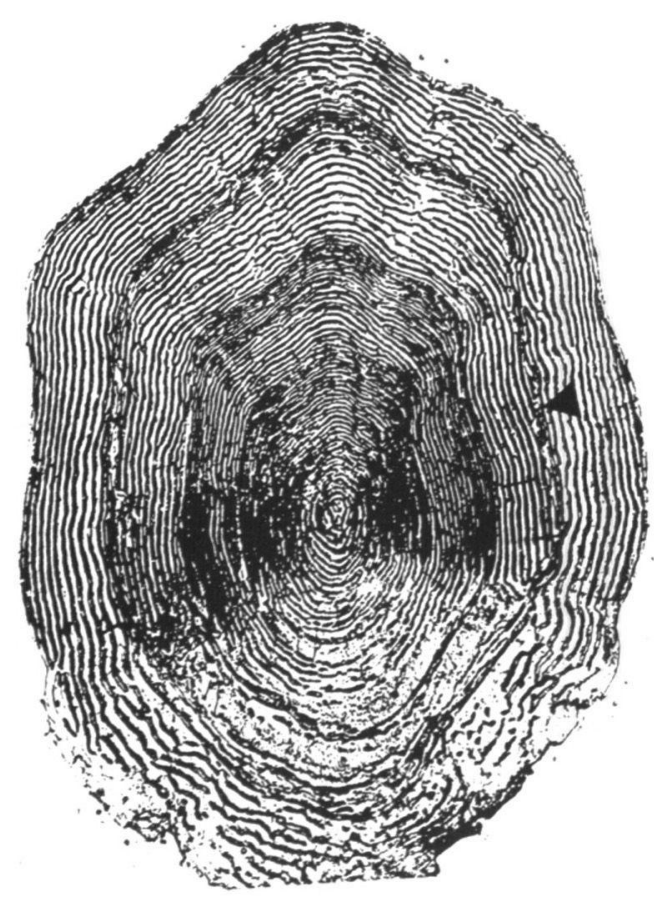

Fig. 25 : Touques, 17.7.84, 475, 2.1+' , marque de reproduction peu prononcée au stade finnock ( 1 ).

Fig. 25 : Touques, 17.7.84, 475, 2.1+', spawning mark less visible at finnock stage (4). 


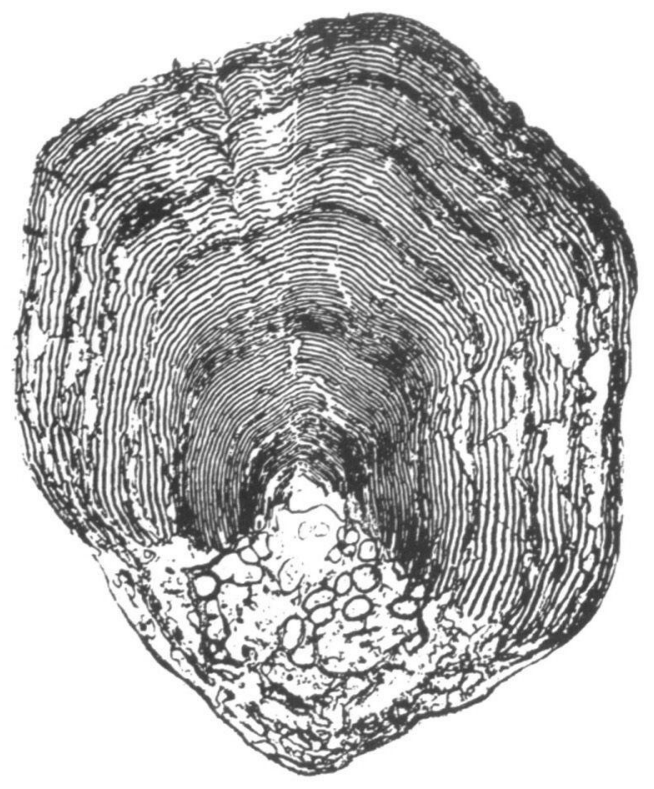

Fig. 26 : Touques, 11.8.83, 560, 2.4+.1234, similitude de la structure des marques de frai successives chez un individu de plusieurs reproductions.

Fig. 26 : Touques, $11.8 .83,560,2.4++^{1234}$, the structure of successive spawning marks is similar in a multi-spawning fish.

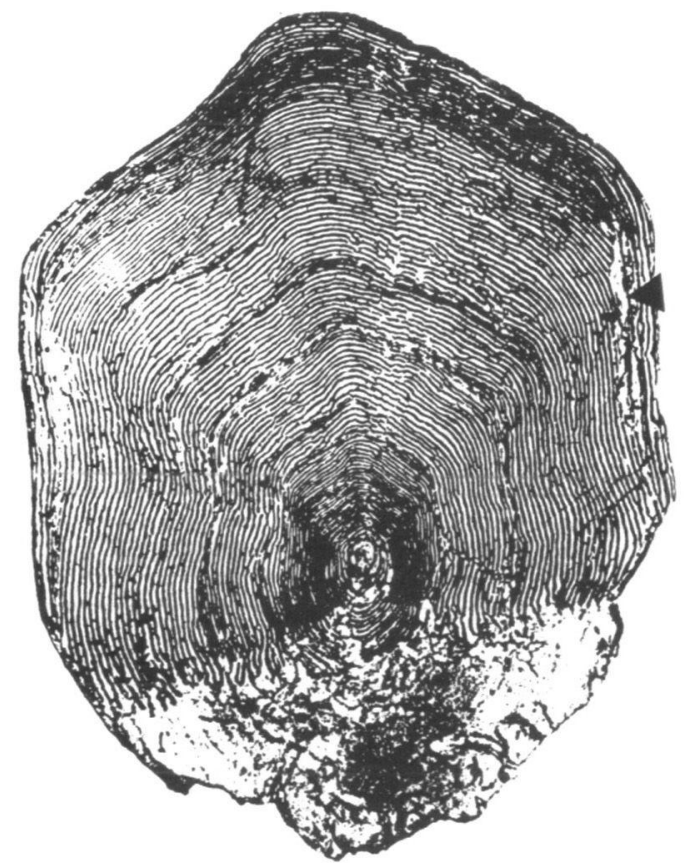

Fig. 28 : Orne, 1.7.83, 684, 1.2+, pseudomarque de frai sur le deuxième hiver marin $(1)$.

Fig. 28 : Orne, 1.7.83, 684, 1.2+, a false spawning mark on the second winter band (४).

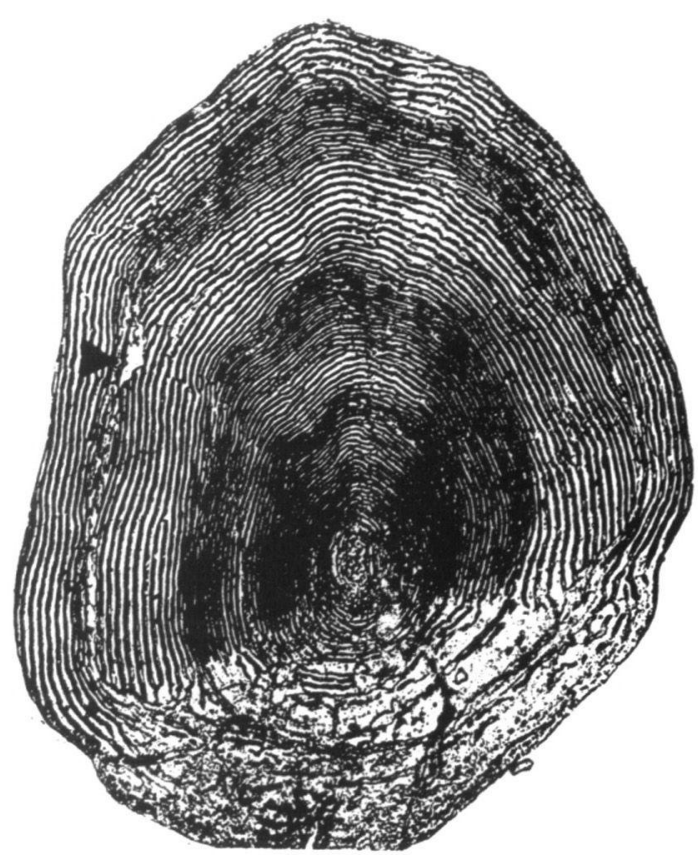

Fig. 27 : Touques, 24.5.84, 526, 2.1t, pseudomarque de frai sur le premier hiver marin ( 4 ).

Fig. 27 : Touques, 24.5.84, 526, 2.1+, a false spawning mark on the first sea winter band ( 4 ).

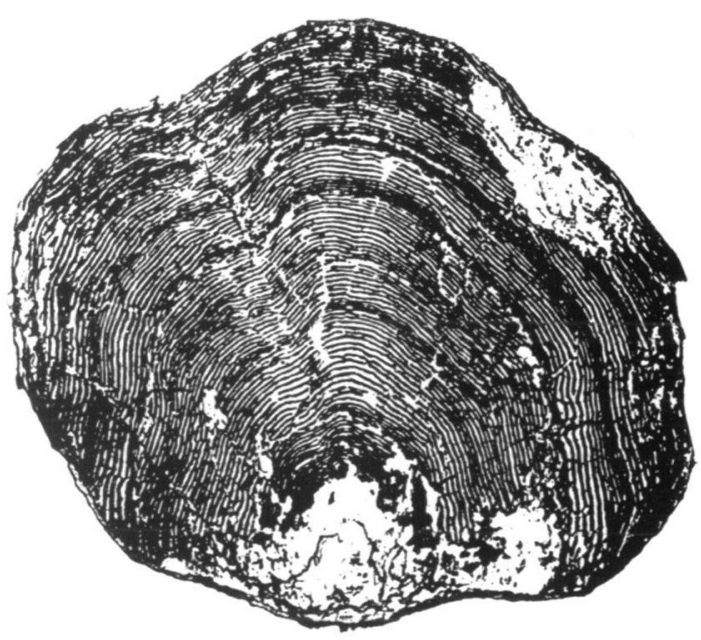

Fig. 29 : Touques, $21.1 .84,750,1.6+23456$, importante érosion superficlelle de l'écaille chez un poisson âgé.

Fig. 29 : Touques, 21.1.84, 750, 2.6+23466 , large erosion on the surface scale in a old fish. 


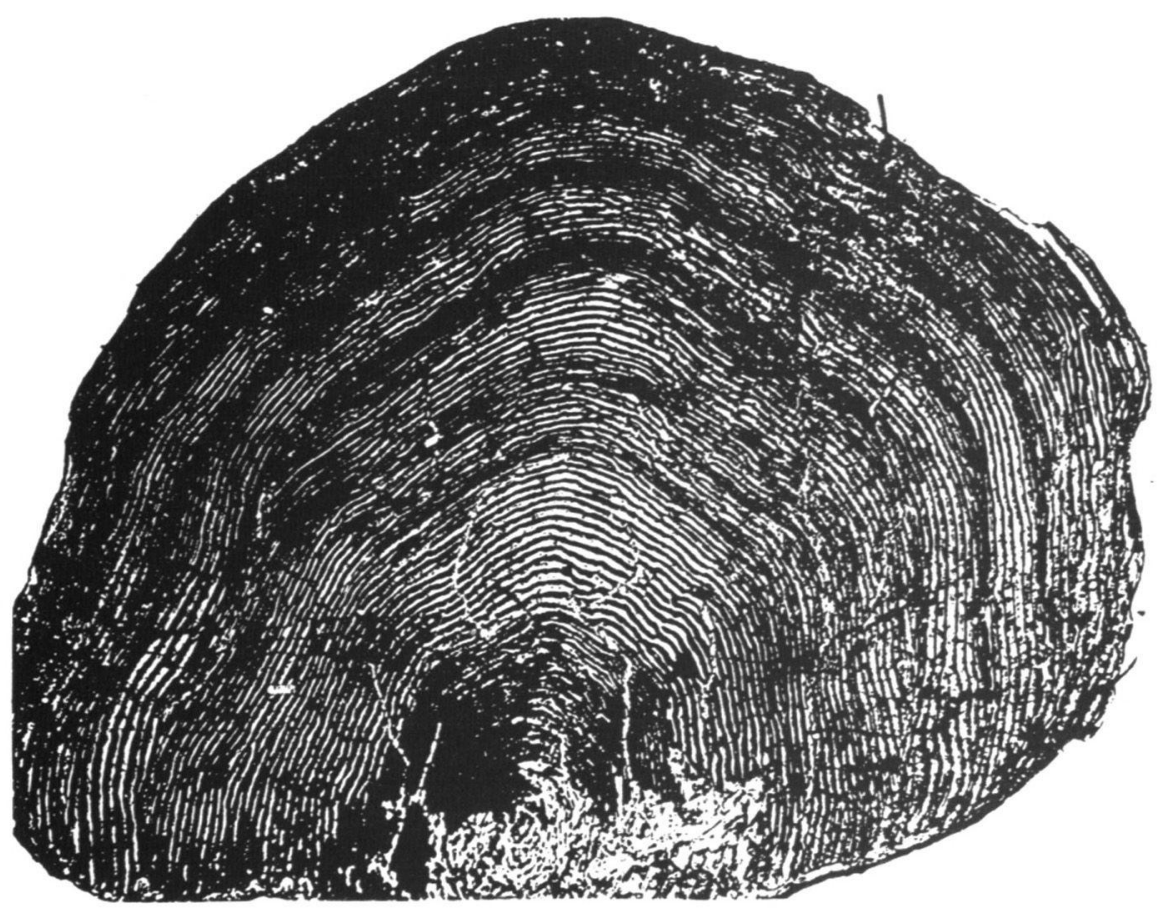

Fig. 30 : Touques, 29.1.84, 750, $1>5+^{2345}$, tassement et recouvrement des marques de frai en bordure de l'écaille chez un poisson âgé. Le nombre de reproductions et l'âge de mer ne peuvent pas être donnés avec précision.

Fig. 30 : Touques, 29.1.84, 750, $1>5+^{2345}$, compression and cutting over of spawning marks on the margin of scale in a old fish. The number of spawnings and the sea age cannot be determinated precisely.

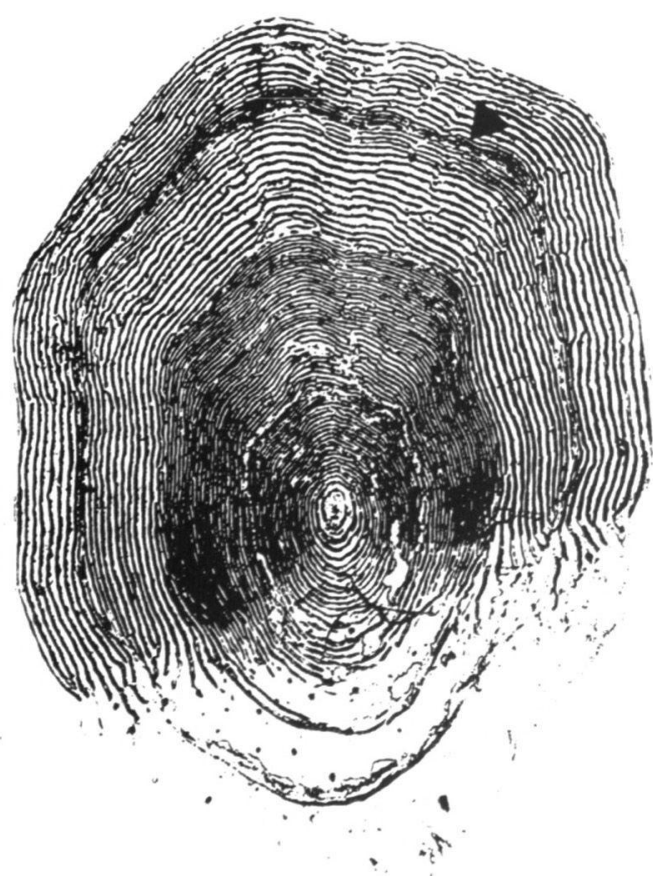

Fig. 31a : Vue générale de la structure.

Fig. 31a : General view of the structure.

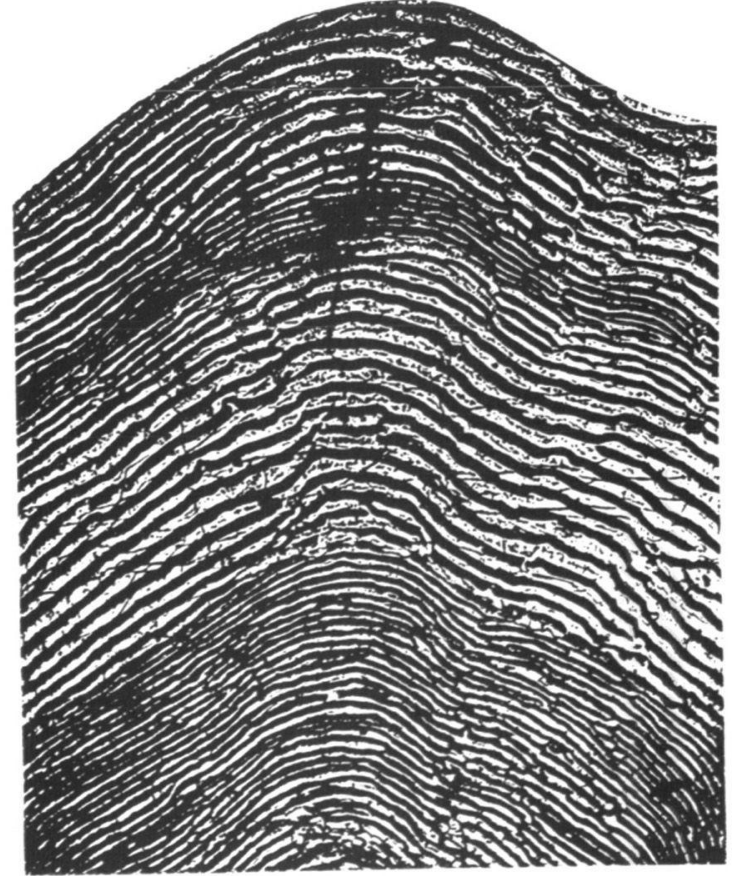

Fig. 31b : Détail de cette structure. Fig. 31b : Detail of the structure.

Fig. 31 : Touques, $9.6 .87,455,1.1 .+^{\mathrm{SF}}$, bande hivernale post dévalaison contituée de nombreuses stries fines $(n>5)(4)$.

Fig. 31 : Touques, $9.6 .87,455,1.1 .+^{F C}$, winter band formed with many fine circuli $(n>5)(4)$ after smolt migration. 


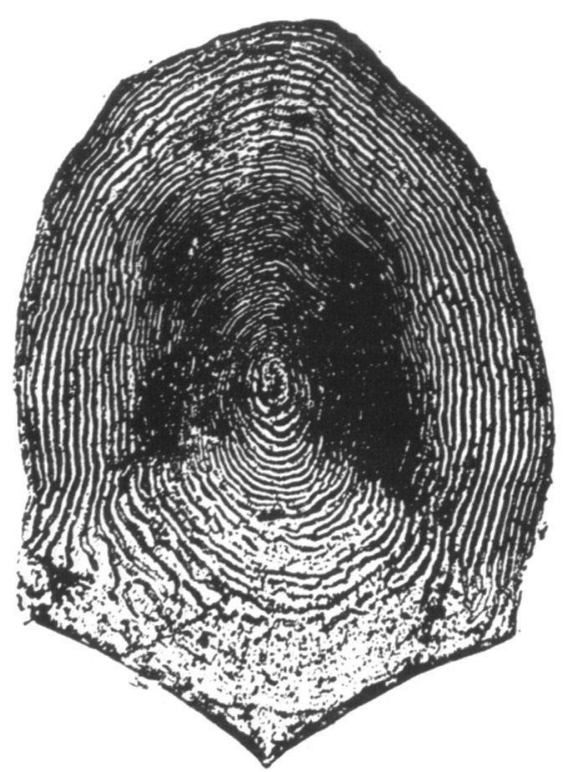

Fig. 32a : Touques, 20.8.83, 305, 1.0+, capture au stade finnock lors d'une première remontée en rivière.

Fig. 32a : Touques, 20.8.83, 305, 1.0+, capture at finnock stage during a first upstream migration in river

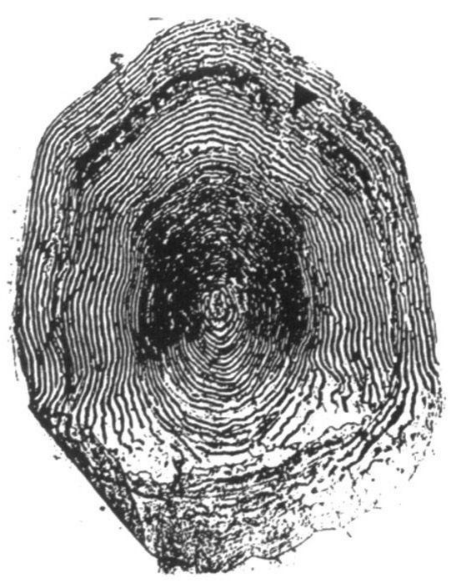

Fig. 32b : Touques, $19.6 .84,418,1.1+^{\mathrm{SF}}$, recapture à l'áge 1+ de mer lors de la migration de reproduction en rivière apres formation d'une bande hivernale à stries fines et nombreuses (4).

Fig. 32b : Touques, $19.6 .84,418,1.1+{ }^{\mathrm{FC}}$, recapture at $1+$ sea age during the spawning migration in river with presence of a previous winter band with many fine circuli $(4)$ on the scale.

Fig. 32 : Validation de la structure bande hivernale post dévalaison à stries fines.

Fig. 32 : Validation of the structure : winter band with many fine circuli after smolt migration.

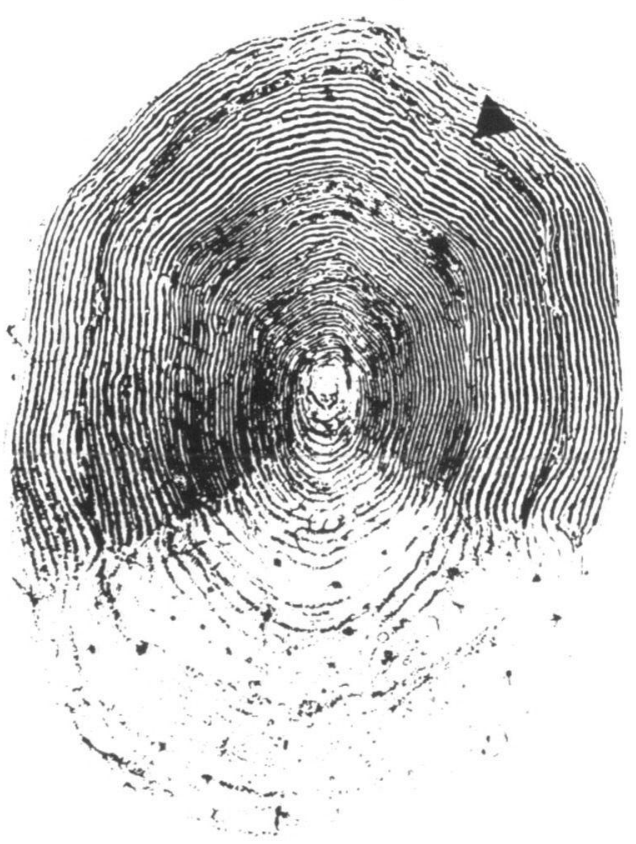

Fig. 33a : Structure sans érosion latérale.

Fig. 33a : Structure without lateral erosion.

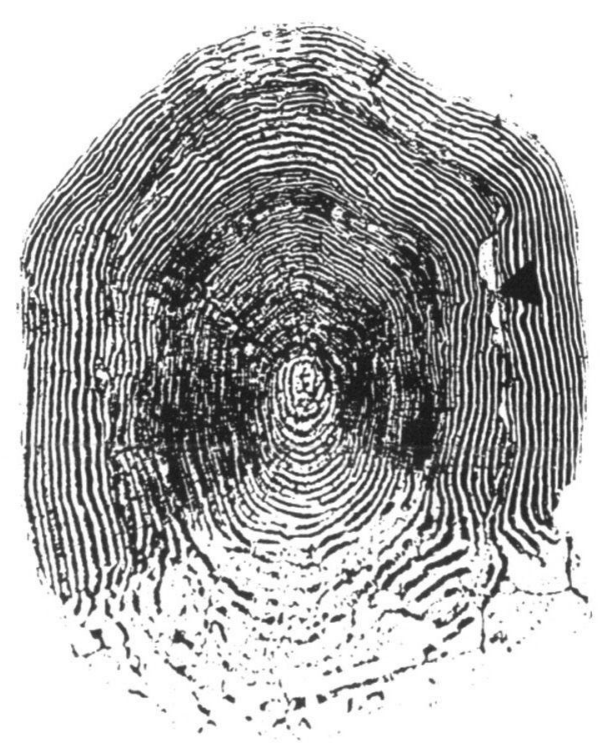

Fig. 33b : Structure avec érosion latérale.

Fig. 33b : Structure with lateral erosion.

Fig. 33 : Touques, $7.7 .87,382,2.1+{ }^{\mathrm{SF}}$, bande hivernale post dévalaison à stries fines peu nombreuses $(n<5)(4)$.

Fig. 33 : Touques, $7.7 .87,382,2.1+^{\mathrm{FC}}$, winter band formed with few fine circuli $(n<5)(4)$ after smolt devalaison. 


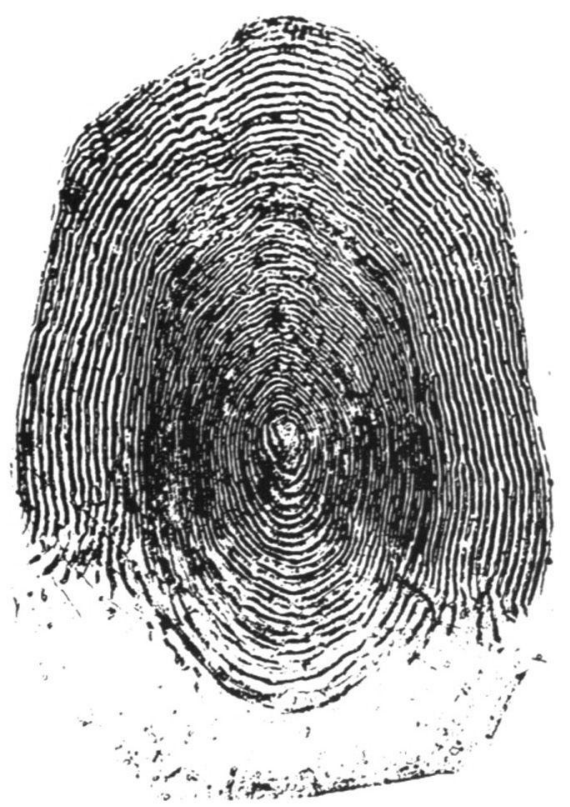

Fig. 34a : Touques, $30.7 .83,317,1.0+$, capture au stade finnock lors d'une première remontée en rivière.

Fig. 34a : Touques, 30.7.83, 317, 1.0+, capture at the finnock stage during a first upstream migration in river.

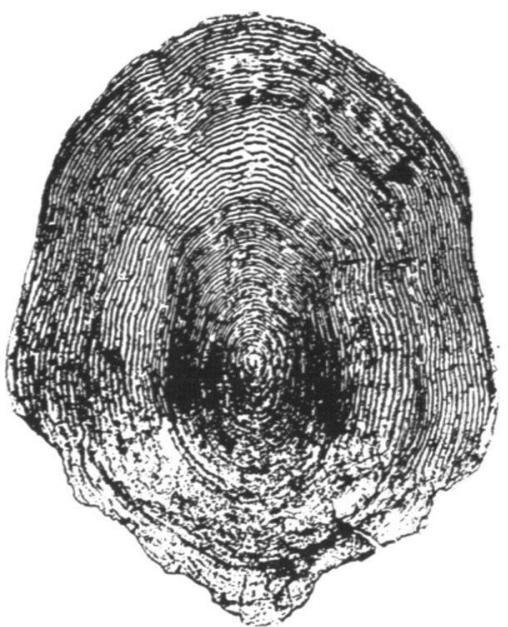

Fig. $34 \mathrm{~b}$ : Touques, $10.7 .84,508,1.1+{ }^{\mathrm{SF}}$, recapture à l'âge 1+ de mer lors de la migration de reproduction en riviere après formation d'une bande hivernale peu épaisse à stries fines $(4)$.

Fig. 34b : Touques, 10.7.84, 508, 1.1+ ${ }^{F C}$, recapture at $1+$ sea age during the spawning migration in river with presence of a previous winter band with few fine circuli $($ ) on the scale.

Fig. 34 : Validation de la structure bande hivernale post dévalaison à stries fines peu nombreuses. Fig. 34 : Validation of the structure : winter band with few fine circuli after smolt migration.

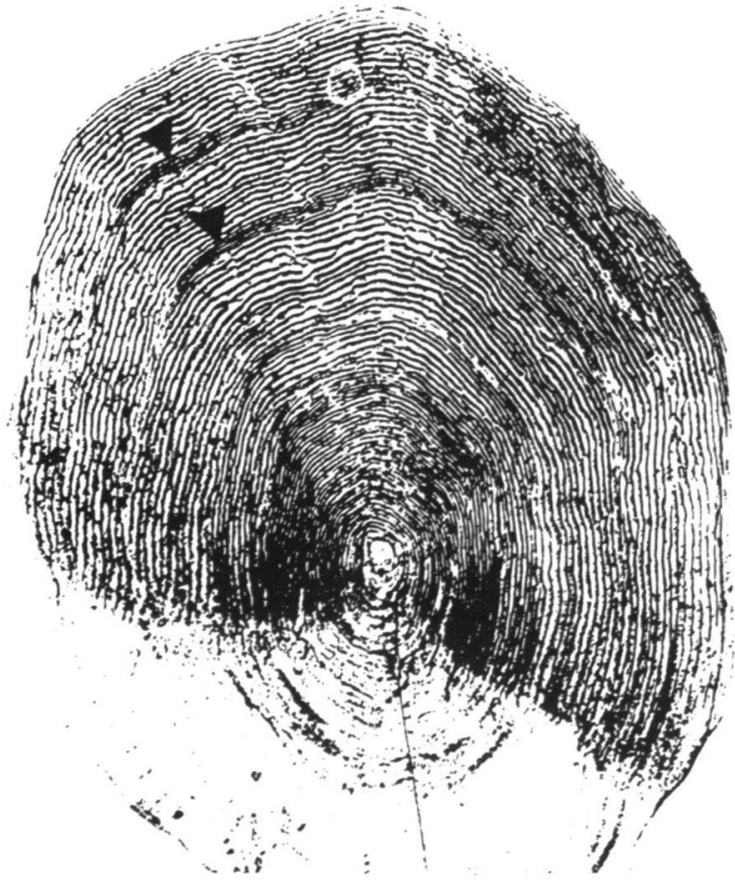

Fig. 35a : Vue générale de la structure.

Fig. 35a : General view of the structure.

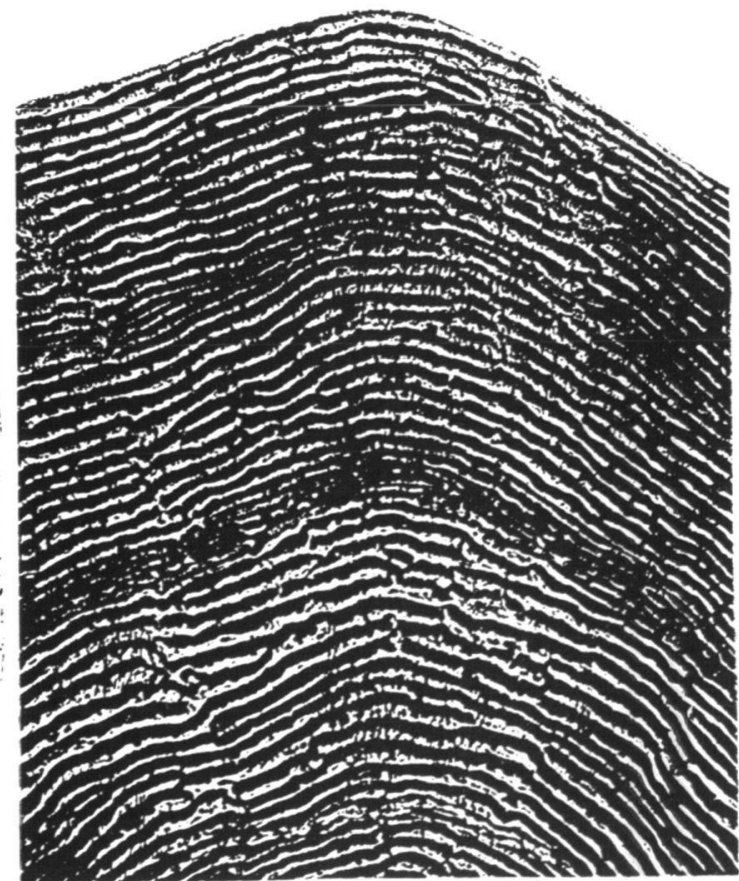

Fig. 35b : Detail de cette structure.

Fig. 35b : Detail of the structure.

Fig. 35 : Touques, 22.6.84, 437, 1.1 $+^{\mathrm{OA}}$, présence d'un double resserrement lors de l'hiver post dévalaison ( $)$.

Fig. 35 : Touques, 22.6.84, 437, 1.1 ${ }^{\mathrm{DB}}$, presence of a double band with narrow-spaced fine circuli after smolt migration $(1)$. 


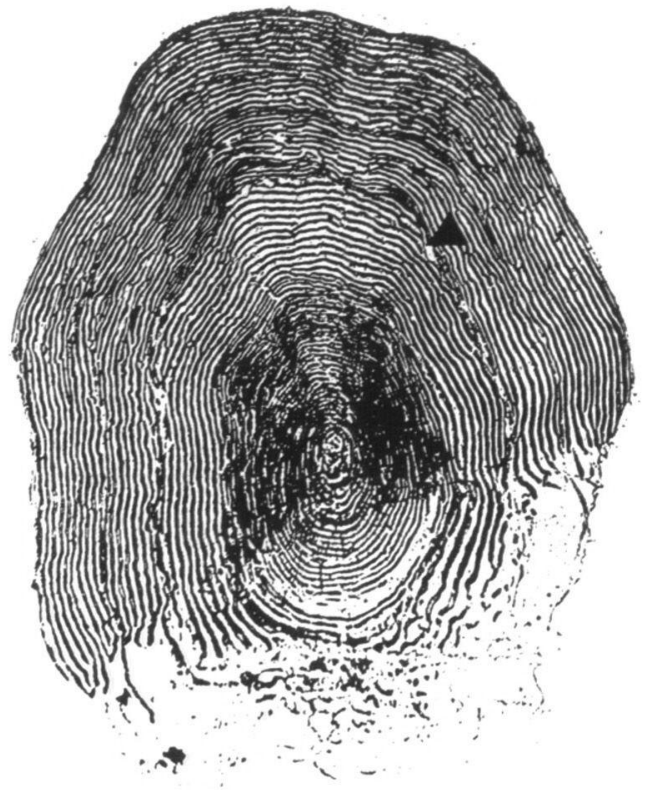

Fig. 36 : Touques, $26.6 .87,437,1.1+{ }^{\mathrm{DR}}$, double resserrement lors de l'hiver post dévalaison, présence d'une usure latérale sur le premier $(4)$.

Fig. 36 : Touques, $26.6 .87,437,1.1+^{\mathrm{DB}}$, double band with narrow-spaced fine circuli after smolt migration, presence of a lateral erosion on the first one $(4)$.

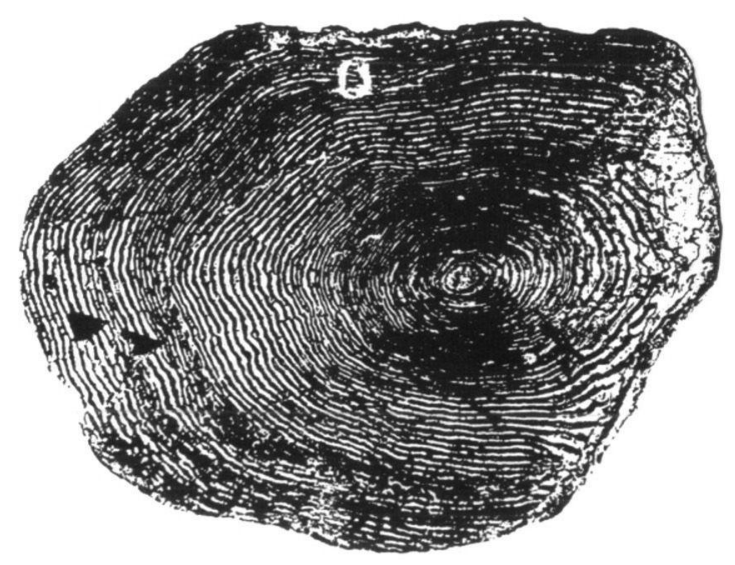

Fig. 37b : Touques, 5.10.84, $463,1.1 .+^{\mathrm{DH}}$, recapture à l'âge 1+ de mer après formation du double resserrement post dévalaison (4).

Fig. 37b : Touques, $5.10 .84,463,1.1 .+^{\mathrm{DB}}$, recapture at $1+$ sea age with presence of a double band of narrow-spaced fine circuli on the scale $(\varangle)$.

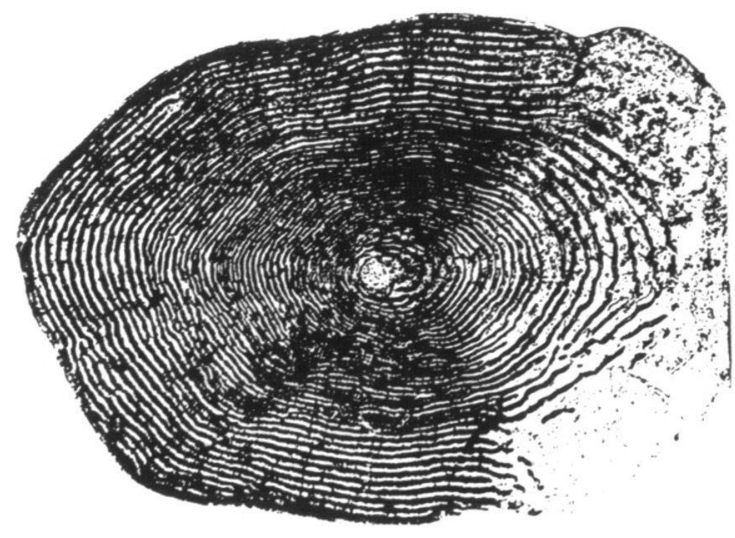

Fig. 37a : Touques, $1.8 .83,307,1.0+$, capture au stade finnock lors d'une première remontée en rivière.

Fig. 37a : Touques, 1.8.83, 307, 1.0+, capture at finnock stage during a first upstream migration in river.

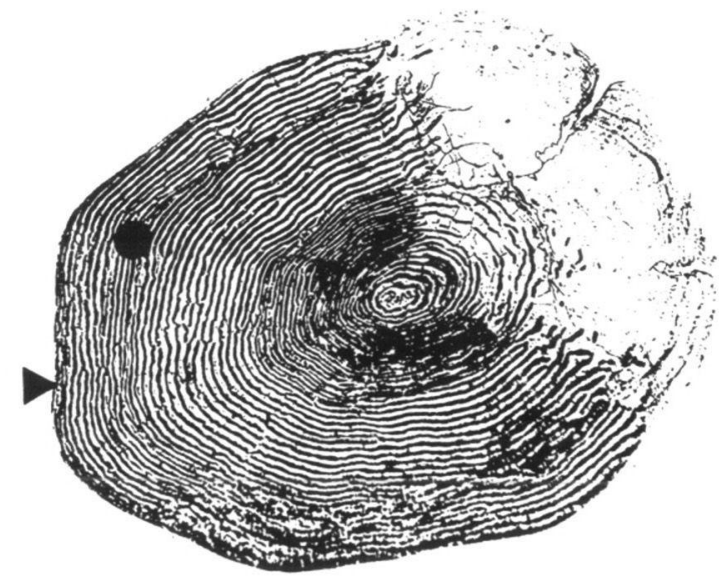

Fig. 37c : Touques, 9.3.84, 340, 1.1, formation du deuxième resserrement $(\checkmark)$ lors d'un deuxième séjour en eau douce ou en estuaire, première bande $(\bullet)$.

Fig. 37c : Touques, 9.3.84, 340, 1.1, beginning of the second band on the scale edge $(\checkmark)$ during a second travel in river or estuary, first band $(\bullet)$.

Fig. 37 : Validation de la structure double resserrement post dévalaison.

Fig. 37 : Validation of the structure : double band with narrow-spaced fine circuli after smolt migration. 


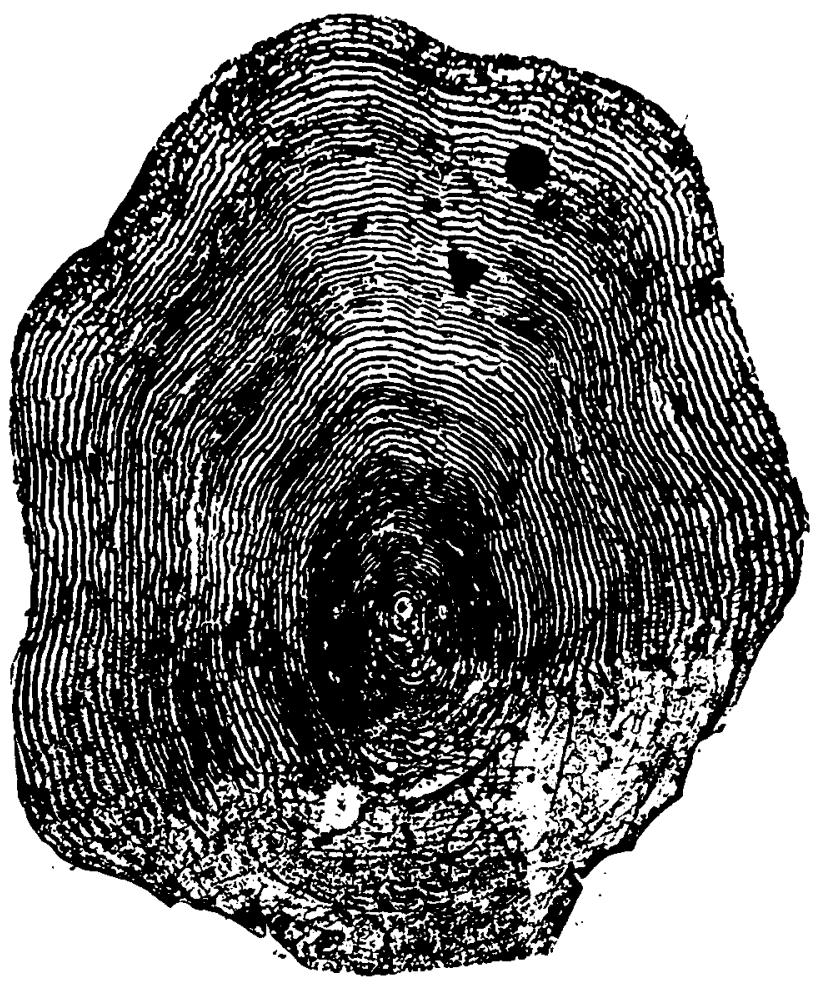

Fig. 38a : Vue générale de la structure.

Fig. 38a : General view of the structure.

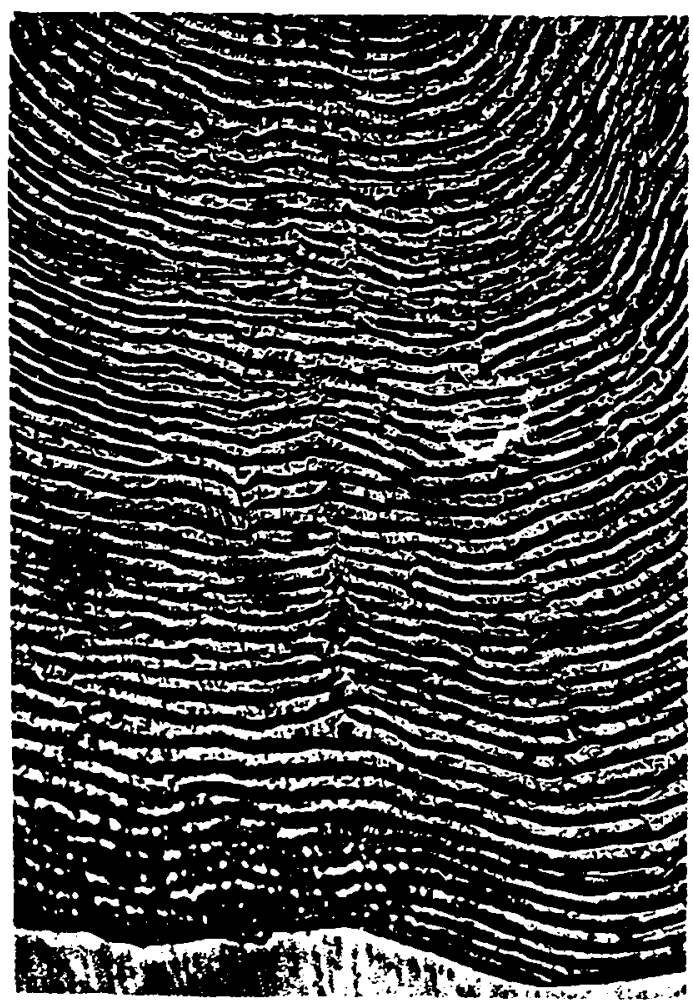

Fig. 38b : Détail de cette structure. Fig. 38b : Detail of the structure.

Fig. 38 : Orne, 5.7.83, 544, 1.1+, double resserrement lors de l'hiver post dévalaison. La première bande est constituée de stries fines $(\downarrow)$, la seconde correspond a un hiver marin $(0)$.

Fig. 38 : Orne, 5.7.83, 544,1.1+, double band after smolt migration. The first one is formed with some narrow-spaced circuli $(4)$, the second one corresponds to a sea winter band $(\Theta)$. 